\title{
RDUS
}

Revue de DROIT

UNIVERSITÉ DE SHERBROOKE

Tiłre : $\quad$ LES AMBIGUIITÉS DU PROCESSUS DISCIPLINAIRE APPLICABLE AUX JUGES DE NOMINATION FÉDÉRALE

Auteur(s): $\quad$ LUc HUPPÉ

Revue : $\quad$ RDUS, 2008-2009, volume 39, numéro 1-2

Pages: $\quad 71-114$

ISSN : $\quad 0317-9656$

Éditeur : $\quad$ Université de Sherbrooke. Faculté de droit.

URI : $\quad$ http://hdl.handle.net/11143/11536

DOI : $\quad$ https://doi.org/10.17118/11143/11536 
Page vide laissée intentionnellement. 


\section{LES AMBIGUÏTÉS DU PROCESSUS DISCIPLINAIRE APPLICABLE AUX JUGES DE NOMINATION FÉDÉRALE}

par Luc HUPPÉ*

Établi par la Loi sur les juges il y a près de quarante ans, le mécanisme de plainte à l'égard des juges de nomination fédérale n'a jusqu'à présent donné lieu qu'à une dizaine d'enquêtes publiques relativement à des inconduites judiciaires. Cette situation peut s'expliquer par l'existence de nombreuses étapes devant être franchies par le Conseil canadien de la magistrature avant qu'un comité d'enquête ne soit constitué. De plus, l'absence d'un encadrement législatif précis complique la mise en ouvre de la déontologie judiciaire au niveau fédéral, notamment quant à la sanction des inconduites mineures. Le présent texte soulève quelques interrogations quant à l'efficacité réelle de ce mécanisme, tel qu'il existe actuellement, par rapport aux attentes légitimes des justiciables envers les institutions judiciaires et leurs membres.

Altough the Judges Act allows any person to file a complaint in respect of a judge appointed by the federal governement, only a few public inquiries have been conducted by the Canadian Judicial Council concerning judicial misconduct since this process has been created almost forty years ago. This situation can be explained by the lenghty procedure that must be followed before an Inquiry Committee may be constituted. Moreover, the absence of legislative provisions regulating judicial conduct at the federal level creates many problems in the enforcement of judicial discipline, particularly in relation to misconduct of a minor nature. This paper raises questions about the ability of this procedure, as it presently stands, to maintain public confidence in the judiciary.

* Avocat, De Grandpré Joli-Cœur, Montréal. 


\section{SOMMAIRE}

INTRODUCTION 73

1. La décision de tenir une enquête publique .............. 76

1.1. Le processus de traitement des plaintes .......... 77

1.2. Les conséquences de ce processus ................ 85

2. La sanction de l'inconduite judiciaire ................... 92

2.1. L'assise des obligations déontologiques ........... 93

2.2. Les formes de l'intervention disciplinaire ......... 99

CONCLUSION $\quad$................................................... 108

ANNEXE : LISTE DES RAPPORTS D'ENGUÊTE ............... 112 
Les ambiguïtés $d u$

(2008-09) 39 R.D.U.S. processus disciplinaire applicable

\section{INTRODUCTION}

Établi en $1971^{1}$ alors que la destitution du juge Landreville était à l'étude ${ }^{2}$, le processus d'examen de la conduite des juges de nomination fédérale a jusqu'à présent suscité quelques milliers de plaintes ${ }^{3}$. C'est au Conseil canadien de la magistrature, constitué par la Loi sur les juges ${ }^{4}$, qu'incombe la responsabilité de recevoir et de traiter ces plaintes ${ }^{5}$. Composé des membres les plus éminents de la magistrature canadienne ${ }^{6}$, le Conseil comprend les juges en chef de tous les tribunaux dont les membres sont assujettis à sa compétence d'enquête, ainsi que les juges en chefs associés et les juges en chef adjoints de ces tribunaux, lorsqu'il en existe. C'est donc dire qu'il regroupe, au sein des institutions judiciaires les plus importantes du pays, ceux des juges dont les

1. Loi modifiant la Loi sur les juges et la Loi sur l'administration financière, (1970-71-72) 19-20-21 Élis. II, c. 55, art. 11; cette disposition est entrée en vigueur le 9 décembre 1971: DORS/71-609. Pour un exposé des différents modèles utilisés à l'égard des juges de nomination provinciale, voir: Thomas Cromwell, "Analyse préliminaire de la procédure disciplinaire relative à la conduite des juges nommés par les provinces", (1998) 22 (1) J.J. prov. 39.

2. À propos des différentes phases de cette affaire, voir : Landreville c. La Reine, [1977] 2 C.F. 726 [Landreville]. Ce jugement a notamment confirmé que le gouvernement fédéral peut autoriser une enquête à propos de la conduite des juges des tribunaux supérieurs (p. 745-746); au même effet, voir aussi : Décision du comité d'enquête en vertu des paragraphes 63 (2) et 63 (3) de la Loi sur les juges relativement à M. le juge F.L. Gratton de la Cour de l'Ontario (division générale), 26 janvier 1994 (C.E.), p. 17 (décision aussi publiée à : (1995) 5 R.N.D.C. 215).

3. Le Conseil canadien de la magistrature mentionne chaque année dans son Rapport annuel (le premier à être rendu public étant celui de 19871988) le nombre de plaintes reçues au cours de l'année écoulée. Ayant graduellement augmenté pour atteindre un maximum de 202 en 19971998, le nombre de plaintes a diminué de plus de $25 \%$ au cours de l'année suivante. Depuis, il a varié entre 138 (pour l'année 2003-2004) et 193 (pour l'année 2006-2007).

4. Loi sur les juges, L.R.C. (1985) c. J-1 [Loi sur les juges].

5. Ibid., art. 60 (2) c).

6. Ibid., art. 59. 
Les ambigütés $d u$

processus disciplinaire applicable (2008-09) 39 R.D.U.S. aux juges de nomination fédérale

fonctions comportent les plus grandes responsabilités dans la mise en œuvre de la déontologie judiciaire? ${ }^{7}$.

Sont notamment assujettis à la compétence d'enquête du Conseil canadien de la magistrature les juges des tribunaux supérieurs de première instance et d'appel, de même que les juges de la Cour fédérale, de la Cour d'appel fédérale et de la Cour suprême du Canada ${ }^{8}$. Les dispositions de la Constitution canadienne ou de la législation fédérale garantissent à ces juges le droit de demeurer en fonction durant bonne conduite jusqu'à l'âge de la retraite ${ }^{9}$. Ils ne peuvent être destitués par le gouverneur général ou le gouverneur en conseil, selon le cas, que sur une adresse du Sénat et de la Chambre des communes.

7. Dans l'arrêt Ruffo c. Conseil de la magistrature, [1995] 4 R.C.S. 267, aux pp. 301-304 (par. 52-59), la Cour suprême du Canada a mis en lumière les responsabilités inhérentes au statut de juge en chef en ce qui a trait à la déontologie judiciaire. À propos de l'encadrement juridique de la déontologie judiciaire au Canada, voir : Luc Huppé, "Les fondements de la déontologie judiciaire", (2004) 45 C. de D. 93 [Huppé].

8. L'article 96 de la Loi constitutionnelle de 1867, (1867) 30-31 Vict. c. 3 (R.U.), accorde au gouverneur général du Canada le pouvoir de nommer les juges des cours supérieures dans chaque province. Les juges de la Cour fédérale et de la Cour d'appel fédérale sont nommés par le gouverneur en conseil en vertu de l'article 5.2 de la Loi sur les Cours fédérales, L.R.C. (1985) c. F-7. Ceux de la Cour suprême du Canada sont aussi nommés par le gouverneur en conseil, aux termes de l'article 4 de la Loi sur la Cour suprême, L.R.C. (1985), c. S-26.

9. Cette garantie est accordée aux juges des tribunaux supérieurs des provinces par l'article 99 de la Loi constitutionnelle de 1867, ibid., note 8. L'inamovibilité des juges de la Cour fédérale et de la Cour d'appel fédérale est consacrée à l'article 8 de la Loi sur les Cour fédérales, ibid., note 8; le bénéfice des dispositions constitutionnelles applicables aux juges des tribunaux provinciaux supérieurs leur a d'ailleurs été rendu applicable par une décision de première instance de la Cour fédérale, qui n'a pas été portée en appel : Addy c. La Reine, [1985] 2 C.F. 452 (1 ère inst.). En ce qui concerne les juges de la Cour suprême du Canada, cette garantie est formulée à l'article 9 de la Loi sur la Cour suprême, supra note 8, une disposition vraisemblablement visée par l'article 42 (1) d) de la Loi constitutionnelle de 1982, constituant l'annexe B de la Loi de 1982 sur le Canada (R.-U.) 1982, c. 11, qui assujettit à la formule générale d'amendement constitutionnel toute modification de la Constitution du Canada portant sur la Cour suprême du Canada. 
Les ambiguïtés $d u$

(2008-09) 39 R.D.U.S. processus disciplinaire applicable

aux juges de nomination fédérale

En dépit de la quantité de plaintes reçues, un nombre très limité d'enquêtes publiques ont été tenues par le Conseil. Tout au plus une dizaine de comités d'enquête constitués aux termes du processus établi par la Loi sur les juges ont eu l'occasion de produire un rapport analysant une plainte d'inconduite judiciaire 10 : quatre rapports d'enquête datent des années 2000 et, pour la période précédente, on compte une moyenne de deux rapports d'enquête par décennie. Si l'on considère que les juges assujettis à ce processus se comptent par centaines dans l'ensemble du pays, de telles enquêtes représentent donc encore un événement exceptionnel au sein de la magistrature.

Depuis que les rapports d'enquête sont rendus publics, trois comités d'enquête seulement ont recommandé la destitution d'un juge. Dans le premier cas, un juge avait fait savoir aux membres d'un jury qu'il considérait leur verdict déraisonnable; il avait aussi tenu publiquement des propos faisant douter de son impartialitél1. Dans le deuxième cas, l'inconduite du juge comportait une participation active aux démarches de citoyens s'opposant à un projet municipal, ainsi que le défaut de prendre les mesures requises pour éviter les conflits d'intérêts que de telles activités pouvaient engendrer ${ }^{12}$; le Conseil canadien de la magistrature a cependant refusé d'entériner la recommandation du comité d'enquête ${ }^{13}$. Dans le troisième cas, le juge s'était livré à de nombreux abus de pouvoir au cours d'un procès criminel et sa conduite donnait lieu à une apparence de partialité envers l'accusé14.

Une étude de l'encadrement législatif et réglementaire du processus de traitement des plaintes concernant les juges de nomination fédérale, ainsi que du contentieux auquel il a donné lieu devant les tribunaux judiciaires, permet de relever deux

10. La liste de ces rapports est reproduite en annexe, avec une indication de la façon dont ils sont cités dans le présent texte.

11. Rapport Bienvenue (C.E.).

12. Rapport Matlow (C.E.).

13. Rapport Matlow (C.C.M.)

14. Rapport Cosgrove (C.E.). 
Les ambiguïtés $d u$

processus disciplinaire applicable (2008-09) 39 R.D.U.S. aux juges de nomination fédérale

éléments plus particulièrement problématiques. En premier lieu, le Conseil canadien de la magistrature pose de nombreuses contraintes - peut-être excessives - à la décision de tenir une enquête publique à propos d'une plainte formulée contre un juge assujetti à sa compétence. En second lieu, la méthode utilisée par le Conseil pour sanctionner l'inconduite judiciaire appelle certaines réserves. L'analyse de ces questions permettra de prendre la mesure de l'efficacité réelle du mécanisme mis en place par la Loi sur les juges pour traiter les écarts de conduite au sein de la magistrature de nomination fédérale.

\section{La décision de tenir une enquête publique}

Hormis les cas où la Loi sur les juges l'y oblige ${ }^{15}$, le Conseil canadien de la magistrature possède une entière discrétion pour enquêter à propos des plaintes qu'il reçoit ${ }^{16}$. Il n'a donc aucune obligation de tenir une enquête publique ${ }^{17}$. Dans ce contexte, l'exercice de la discrétion qui lui est accordée devient un enjeu juridique de première importance, car l'effectivité du processus d'enquête établi par la Loi sur les juges en dépend. Le Conseil s'est donné des lignes directrices pour encadrer cette discrétion : les Procédures relatives à l'examen des plaintes déposées au Conseil canadien de la magistrature au sujet de juges de nomination fédérale ${ }^{18}$. Elles exposent un processus complexe qui,

15. Voir infra, note 37.

16. Loi sur les juges, supra note 4, au par. 63 (2). La Cour d'appel fédérale a noté que cette disposition ne précise pas les critères régissant l'exercice du pouvoir discrétionnaire accordé au Conseil : Taylor c. Canada (Procureur général), [2003] 3 C.F. 3 (C.A.), à la p. 31 (par. 60); autorisation de pourvoi à la Cour suprême du Canada refusée : [2003] 2 R.C.S. xi.

17. Taylor c. Canada (Procureur général), [2002] 3 C.F. 91 (1ière inst.), à la p. 123 (par. 63); jugement confirmé par Taylor c. Canada (Procureur général), supra note 16.

18. Ci-après "Procédures". Entrées en vigueur le 1er janvier 2003, elles ont été publiées dans le Rapport annuel du Conseil de la magistrature, 20022003, p. 49. Ces Procédures reprennent la structure auparavant exposée dans le Règlement administratif $d u$ Conseil, dont on peut suivre l'évolution dans les rapports annuels du Conseil à compter de l'exercice 1989-1990 jusqu'à l'exercice 2002-2003. Le Conseil possède le pouvoir 
Les ambiguïtés $d u$

(2008-09) 39 R.D.U.S. processus disciplinaire applicable

aux juges de nomination fédérale

s'il permet un traitement nuancé et délicat des plaintes reçues par le Conseil, pose néanmoins de lourdes contraintes à la tenue d'une enquête publique par un comité d'enquête et entraîne des conséquences importantes pour les participants à ce processus.

\subsection{Le processus de traitement des plaintes}

En vertu de ces Procédures, quatre étapes doivent être franchies avant que le Conseil ne décide de tenir une enquête publique $^{19}$. En premier lieu, le directeur exécutif du Conseil reçoit les plaintes et exerce un premier contrôle de leur recevabilité20; il peut décider de ne pas ouvrir de dossier à l'égard d'une plainte qu'il considère nettement irrationnelle ou qui constitue selon lui

de régir la procédure relative aux enquêtes portant sur la conduite des juges visés par une plainte : Loi sur les juges, supra note 4, art. 61 (3) c).

19. Ces étapes ont été relatées sans commentaires dans : Cosgrove c. Conseil canadien de la magistrature, [2007] 4 R.C.F. 714 (C.A.F.), aux pp. 741742 (par. 66-73) [Cosgrove]; demande d'autorisation d'appel à la Cour suprême du Canada refusée: [2007] 3 R.C.S. x; Akladyous c. Conseil canadien de la magistrature, 2008 CF 50 (C.F. 1ère inst.), au par. 46-52 [Akladyous]. À propos du déroulement de ce processus, voir aussi : R.J. SCOTT, "Accountability and Independence", (1996) 45 R.D. U.N.-B. 27, aux pp. 29-31.

20. Art. 2.2 des Procédures. Aux termes de l'article 3.2 des Procédures relatives au fonctionnement $d u$ Conseil canadien de la magistrature (publiées dans le Rapport annuel du Conseil de la magistrature, 20022003, à la p. 59), le directeur exécutif est l'administrateur principal du Conseil. Aucune disposition de la Loi sur les juges n'autorise toutefois le Conseil canadien de la magistrature à déléguer à une personne qui n'en est pas membre une partie de ses responsabilités dans le traitement des plaintes. Dans Re Therrien, [2001] 2 R.C.S. 3, à la p. 63 (par. 93), la Cour suprême du Canada écrivait d'ailleurs, à propos des dispositions d'une loi provinciale concernant la discipline judiciaire, que l'organisme à qui la loi attribue l'exercice d'un pouvoir doit l'exercer lui-même et qu'il ne peut le déléguer à un ou plusieurs de ses membres sans y être autorisé par la loi. La seule possibilité de délégation prévue par la Loi sur les juges, au paragraphe 61 (3), existe en faveur des comités constitués par le Conseil. C'est sans doute pour cette raison que l'article 2.1 des Procédures précise que le directeur exécutif agit comme auxiliaire, sous la direction du président du Comité sur la conduite des juges, pour toutes questions liées aux fonctions qu'exerce le Conseil à l'égard des plaintes. 
Les ambiguïtés $d u$

processus disciplinaire applicable (2008-09) 39 R.D.U.S. aux juges de nomination fédérale

un abus manifeste de la procédure relative aux plaintes ${ }^{21}$. Lorsque le directeur exécutif ouvre un dossier à propos d'une plainte, elle est examinée par le président ou un vice-président d'un comité du Conseil, le Comité sur la conduite des juges ${ }^{22}$. Celui-ci peut fermer le dossier s'il estime que la plainte est frivole ou vexatoire, qu'elle est formulée dans un but injustifié, qu'elle est manifestement dénuée de fondement ou qu'elle ne nécessite pas un examen plus poussé ${ }^{23}$. Au terme d'un examen qui peut l'amener à demander des renseignements supplémentaires au plaignant ou à obtenir les commentaires du juge visé par la plainte ou de son juge en chef, il peut aussi fermer le dossier s'il conclut que la plainte est dénuée de fondement ou qu'elle ne nécessite pas un examen plus poussé24.

Mais le dossier peut aussi être fermé dès la deuxième étape pour d'autres raisons. Ce peut être le cas lorsque le juge visé par la plainte reconnaît que sa conduite était déplacée et que le

21. Dans Akladyous, supra note 19, au par. 20, on trouve un exemple du type de raisons invoquées par le directeur exécutif pour refuser d'ouvrir un dossier.

22. Ce comité est formé aux termes de l'article 7 des Procédures.

23. Art. 3.5 a) des Procédures.

24. Art. 5.1 a) i) et 8.1 a) des Procédures. Selon la Cour d'appel fédérale, le rôle du président ou du vice-président du Comité sur la conduite des juges ne consiste pas uniquement à se demander s'il existe un commencement de preuve justifiant la destitution du juge visé par la plainte: Taylor c. Canada (Procureur général), supra note 16, aux pp. 3031 (par. 59-60). Cet arrêt interprétait l'article 50 (1) b) du Règlement administratif de 1998 (Rapport canadien du Conseil canadien de la magistrature 1999-2000, Annexe D). La disposition correspondante des Procédures de 2003 ne fait pas de lien aussi direct avec la destitution du juge, mais permet au président ou au vice-président de fermer le dossier s'il estime que la plainte "ne nécessite pas un examen plus poussé". La différence de vocabulaire n'altère cependant pas la finalité du processus de traitement de la plainte, qui consiste à décider de la tenue d'une enquête formelle concernant l'opportunité de recommander la destitution du juge. Ainsi, dans la Décision du président du comité sur la conduite des juges concernant le juge Leask, 20 septembre 2007, le président a fermé un dossier après avoir déterminé que la conduite du juge "n'était pas suffisamment grave pour le rendre incapable de continuer à exercer la fonction de juge". 
Les ambiguïtés $d u$

(2008-09) 39 R.D.U.S. processus disciplinaire applicable

aux juges de nomination fédérale

président ou le vice-président du Comité sur la conduite des juges considère qu'aucune mesure autre que cette expression de regret ne paraît nécessaire ${ }^{25}$. Le dossier peut aussi être mis en suspens, en attendant l'application de mesures correctives prises de consentement avec le juge visé par la plainte, puis fermé si le président ou le vice-président du Comité sur la conduite des juges est satisfait que les problèmes relevés par la plainte ont été traités de façon appropriée ${ }^{26}$. Une étude effectuée en 2002 montrait que seulement $3 \%$ des plaintes franchissent cette deuxième étape, qui constitue donc une phase cruciale du processus d'examen des plaintes ${ }^{27}$.

Si le dossier n'est pas préalablement fermé, la troisième étape du processus se déroule devant un sous-comité, composé de trois ou cinq membres, à qui le président ou le vice-président du Comité sur la conduite des juges défère la plainte ${ }^{28}$. Après analyse, ce sous-comité peut fermer le dossier s'il décide qu'aucun comité d'enquête ne devrait être constitué ou mettre le dossier en suspens en attendant l'application de mesures correctives ${ }^{29}$. S'il est d'avis que l'affaire peut être suffisamment grave pour justifier la destitution du juge, il peut recommander au Conseil canadien de la magistrature de constituer un comité d'enquête ${ }^{30}$.

25. Art. 5.1 a) ii) et 8.1 a) des Procédures.

26. Art. 5.1 b), 5.3 et 8.1 b) des Procédures.

27. Conseil canadien de la magistrature, Rapport annuel 2001-2002, à la p. 26. Le Conseil canadien de la magistrature reconnaît d'ailleurs à chaque année dans ses rapports annuels que la plupart des dossiers de plainte sont fermés à cette étape. La Cour d'appel fédérale a aussi noté que la grande majorité des plaintes relevant de la discrétion du Conseil sont rejetées sommairement et que, sur le petit nombre restant, presque toutes sont réglées au moyen de mesures correctives ou d'une lettre d'explications : Cosgrove, supra note 19, à la p. 43 (par. 74); à ce sujet, voir aussi : Décision du comité d'enquête en vertu des paragraphes 63 (2) et 63 (3) de la Loi sur les juges relativement à M. le juge F.L. Gratton de la Cour de l'Ontario (division générale), 26 janvier 1994 (C.E.), à la p. 11.

28. $\quad$ Art. 5.1 d) et 8.1 c) des Procédures.

29. $\quad$ Art. 9.6 b) et c) des Procédures.

30. Art. 9.6 d) des Procédures. On peut ainsi constater que les sous-comités examinent, aux fins du rapport qu'ils remettent au Conseil, le même critère de destitution que celui élaboré par les comités d'enquête au 
En ce cas, dans une quatrième étape, le Conseil doit encore donner au juge l'occasion de présenter des observations écrites expliquant pourquoi une enquête devrait ou ne devrait pas être tenue $^{31}$. C'est à lui qu'incombe, en dernière analyse, la responsabilité de constituer un comité d'enquête, une décision qu'il prend s'il considère que l'affaire est suffisamment grave pour justifier la destitution du juge ${ }^{32}$. Après la tenue de l'enquête publique par un comité d'enquête, c'est aussi au Conseil qu'il incombe de faire ses recommandations au ministre de la Justice du Canada quant à l'opportunité de destituer le juge ${ }^{33}$.

De son dépôt initial jusqu'au rapport final éventuellement adressé par le Conseil canadien de la magistrature au ministre de la Justice, une plainte peut donc faire l'objet de six appréciations distinctes, dont deux par le Conseil canadien de la magistrature en plénière. À chacun de ces stades, le dossier factuel est susceptible de varier en fonction des exigences formulées par chacune des instances successivement saisies de la plainte, qui demeurent entièrement libres de déterminer la nature et l'ampleur des éléments de preuve ou des représentations dont elles considèrent devoir prendre connaissance pour parvenir à une décision.

L'allongement considérable de ce processus, par sa fragmentation en de multiples étapes, semble difficile à justifier si l'on considère le rôle limité du Conseil canadien de la magistrature dans le mécanisme de destitution des juges de nomination fédérale. Le Conseil n'exerce en effet aucune fonction

cours des enquêtes publiques : Décision d'un sous-comité concernant le juge McLung, 19 mai 1999; Décision d'un sous-comité concernant le juge Barakett, 24 juillet 2002.

31. $\quad$ Art. 10.3 des Procédures.

32. $\quad$ Art. 10.4 b) des Procédures.

33. Art. 65 de la Loi sur les juges, supra note 4. À ce stade, et malgré toutes les démarches préalables de ses instances internes, le Conseil peut encore recevoir et entendre d'autres éléments de preuve : Art. 9 et $10 \mathrm{du}$ Règlement administratif du Conseil canadien de la magistrature sur les enquêtes, DORS/2002-371; ce pouvoir n'est pas purement théorique : Rapport Matlow (C.C.M.), au par. 54. 
Les ambiguïtés $d u$

(2008-09) 39 R.D.U.S. processus disciplinaire applicable

décisionnelle à ce sujet ${ }^{34}$ puisque, aux termes des dispositions constitutionnelles applicables, ce pouvoir appartient au gouverneur général ou au gouverneur général en conseil et qu'il est exercé sur une adresse du Sénat et de la Chambre des communes. Ces assemblées parlementaires ne sont pas légalement tenues de donner suite aux recommandations du Conseil canadien de la magistrature. Leurs éventuelles démarches en vue de la destitution d'un juge demeurent indépendantes de la tenue d'une enquête par le Conseil ou du résultat d'une telle enquête ${ }^{35}$. Le processus d'enquête auquel le Conseil se livre débouche sur une recommandation au ministre de la Justice du Canada et, par conséquent, ne remet pas directement en cause l'inamovibilité du juge. Quoique le statut de ses membres donne une grande valeur à l'opinion formulée par le Conseil, on peut donc s'interroger à propos de l'utilité d'un aussi long cheminement de la plainte auprès de ses instances internes.

Il existe un moyen de passer outre aux quatre premières étapes du processus établies par les Procédures : la Loi sur les juges exige que le Conseil canadien de la magistrature fasse enquête lorsque le ministre de la Justice du Canada ou le

34. Gratton c. Conseil canadien de la magistrature, [1994] 2 C.F. 769 (1 ère inst.), p. 800-802 [Gratton]; Taylor c. Canada (Procureur général), [2002] 3 C.F. 91 (1ière inst.), aux pp. 119-120, (par. 49); jugement confirmé par Taylor c. Canada (Procureur général), supra note 16. L'article 73 de la Loi sur les juges, supra note 4, énonce d'ailleurs que le processus d'enquête et de recommandation établi par cette loi ne porte pas atteinte aux attributions de la Chambre des communes, du Sénat ou du gouverneur en conseil relativement à la destitution des juges.

35. Dans Cosgrove c. Conseil canadien de la magistrature, supra note 19, à la p. 735 (par. 49), la Cour d'appel fédérale a confirmé que le pouvoir du gouverneur général de démettre un juge ne dépend pas de la tenue d'une enquête devant le Conseil canadien de la magistrature et qu'un juge peut être destitué même si ce processus n'a pas été suivi; voir aussi: Landreville, supra note 2, à la p. 746; Gratton c. Conseil canadien de la magistrature, supra note 34, à la p. 803. Dans le Rapport Marshall (C.E.), un comité d'enquête a considéré son enquête comme "la première étape $d u$ processus parlementaire canadien de révocation d'un juge" (p. 26), mais cette étape n'est donc pas obligatoire. 
procureur général d'une province le lui demande36. Cette faculté accordée aux autorités gouvernementales de provoquer la tenue d'une enquête à propos d'un juge constitue un contrepoids à la discrétion du Conseil dans le traitement des plaintes. Elle sert de toute évidence à empêcher la paralysie du mécanisme de plaintes établi par la Loi sur les juges. Elle permet aussi d'éviter la constitution, par le gouvernement fédéral, d'une instance ad hoc pour enquêter à propos de la conduite d'un juge.

Il est significatif de constater qu'une bonne part des enquêtes publiques tenues par des comités d'enquête faisaient suite à des demandes adressées au Conseil canadien de la magistrature par le procureur général d'une province ${ }^{37}$. Les faits relatés dans l'un de ces rapports d'enquête démontrent d'ailleurs que la demande du procureur général était le seul moyen d'obtenir la formation d'un comité d'enquête, le président du Comité sur la conduite des juges ayant auparavant par deux fois fermé le dossier d'une plainte déposée par un justiciable à propos des

36. Loi sur les juges, supra note 4, art. 63 (1). La validité constitutionnelle de cette disposition, en ce qui concerne l'obligation du Conseil canadien de la magistrature de tenir une enquête à la demande du procureur général d'une province, a été confirmée par la Cour d'appel fédérale : Cosgrove, supra note 19.

37. Trois rapports d'enquête ont été rendus suite à une demande du procureur général du Québec (le Rapport Bienvenue, le Rapport Flynn et le Rapport Boilard), un à la demande du procureur général de la Nouvelle-Écosse (le Rapport Marshall) et un à la demande du procureur général de l'Ontario (le Rapport Cosgrove). Deux rapports d'enquête faisaient suite à des plaintes déposées par des autorités publiques, soit un juge de la Cour fédérale (le Rapport Berger) et une avocate du contentieux de la Ville de Toronto (le Rapport Matlow). Des comités d'enquêtes ont aussi été constitués à la demande du ministre de la justice du Canada (concernant le juge Flahiff) et du juge en chef de la division générale de la Cour de l'Ontario (concernant le juge Gratton), mais n'ont pas eu à rendre de rapport. Des trois enquêtes formelles tenues en privé et ayant donné lieu à un rapport d'enquête qui n'a pas été publié, l'une avait été demandée par le ministre fédéral de la Justice, une autre par le juge en chef du tribunal dont faisait partie le juge concerné et la troisième par un avocat: Conseil canadien de la magistrature, Rapport annuel 1996-1997, aux pp. 19-20. 
Les ambiguïtés $d u$

(2008-09) 39 R.D.U.S. processus disciplinaire applicable

mêmes faits que ceux dénoncés par le procureur général ${ }^{38}$. Sans cette faculté accordée par la loi de contraindre le Conseil, la tenue d'une enquête publique par un comité d'enquête pourrait donc se produire de manière encore plus rarissime.

Le Conseil canadien de la magistrature considère néanmoins pouvoir passer outre à ce devoir que lui impose la Loi sur les juges. Dans la décision par laquelle il a refusé d'entériner le Rapport Boilard (C.E.), le Conseil a donné à cette obligation légale une interprétation des plus restrictives ${ }^{39}$. Lorsque le ministre de la Justice du Canada ou le procureur général d'une province lui demande de faire enquête à propos de la décision d'un juge, mais sans que la mauvaise foi du juge ou que la commission d'un abus dans l'exercice de ses fonctions ne soient alléguées ou apparentes, le comité d'enquête doit se demander de façon préliminaire s'il existe un élément quelconque permettant de réfuter la présomption de bonne foi du juge. En l'absence d'un tel élément, le comité d'enquête doit refuser de poursuivre son examen de la plainte. S'il procède néanmoins, le Conseil peut luimême exprimer ce refus lorsque le comité d'enquête lui remet son rapport.

Bien qu'il soit nécessaire de prémunir les juges contre le harcèlement ou l'intimidation que pourraient exercer les autorités gouvernementales au moyen du droit qui leur est accordé par la Loi sur les juges de forcer la tenue d'une enquête, la position adoptée par le Conseil dans le Rapport Boilard (C.C.M.) lui permet

38. Rapport Boilard (C.E.), aux pp. 18-22. Ces deux refus du président du Comité sur la conduite des juges donnent un éclairage particulier à la rebuffade subséquemment imposée par le Conseil au comité d'enquête, quant à la décision de tenir l'enquête exigée par le Procureur général.

39. Rapport Boilard (C.C.M.). Le principe alors énoncé par le Conseil a été ultérieurement précisé dans le Rapport Matlow (C.C.M.), aux par. 130131. Il a aussi été évoqué sans désapprobation par la Cour fédérale : Cosgrove, supra note 19, aux pp. 737 et 745 (par. 52 et 80); Cosgrove c. Attorney General of Canada, 2008 FC 941 (1 ère inst.), au par. 16; dans cette dernière affaire, la Cour fédérale a en outre décidé qu'il incombait au Conseil ou au comité d'enquête de déterminer le moment opportun pour décider de l'application de ce principe à l'égard d'une plainte. 
de refuser d'enquêter sans qu'aucun abus de la part du procureur général ou du ministre de la Justice du Canada ne soit démontré. Elle exige en quelque sorte de ceux-ci qu'ils justifient de façon préliminaire leur décision de se prévaloir du droit que leur accorde la Loi sur les juges et qu'ils fassent au Conseil la démonstration de leur propre bonne foi dans l'exercice de ce droit. Un tel obstacle ne peut que contribuer à rendre encore plus inaccessible la compétence d'enquête du Conseil. En outre, si de tels refus se produisaient régulièrement, ils pourraient remettre en cause l'opportunité d'avoir recours à l'expertise du Conseil pour enquêter à propos de la conduite des juges.

Globalement, c'est donc une politique de fermeture qui semble être appliquée par le Conseil canadien de la magistrature quant à la tenue d'une enquête publique à propos d'une plainte ${ }^{40}$. Quelle que soit la forme sous laquelle elle se concrétise, cette politique ne peut avoir qu'un effet dissuasif. Connaissant la probabilité infime que le Conseil décide de tenir une telle enquête s'ils déposent une plainte, ainsi que les multiples occasions fournies par les Procédures de fermer définitivement un dossier sans même qu'ils soient consultés, les justiciables risquent de s'abstenir de dénoncer les inconduites judiciaires dont ils pourraient être témoins. En constatant que même la plainte formulée par le juge en chef d'une province - qui est pourtant membre du Conseil et spécialement chargé par sa fonction de surveiller l'application de la déontologie judiciaire - ne parvient pas à passer l'étape de l'analyse du dossier par le président ou le vice-président du Comité sur la conduite des juges ${ }^{41}$, le public

40. À une certaine époque, le Conseil reconnaissait cette politique en déclarant qu'«à moins qu'elle ne soit d'une rareté exceptionnelle, toute enquête formelle soulèverait des doutes sérieux quant à la valeur du processus de nomination des juges" : Conseil canadien de la magistrature, Rapport annuel 1988-1989, à la p. 13. Bien qu'on ne retrouve plus de tels propos dans les rapports annuels du Conseil, le nombre d'enquêtes publiques demeure encore tout de même très limité.

41. C'est ce qui s'est produit dans la Décision du vice-président du comité sur la conduite des juges concernant le juge Alain, 20 août 2007, malgré que le vice-président ait conclu que la conduite du juge visé par la plainte 
Les ambiguïtés $d u$

(2008-09) 39 R.D.U.S. processus disciplinaire applicable

peut fort légitimement émettre des doutes à propos de l'utilité réelle du mécanisme de plaintes établi par la Loi sur les juges.

\subsection{Les conséquences de ce processus}

La structure de traitement des plaintes mise en place par les Procédures présente de nombreux écueils pour le plaignant. À la différence du juge visé par la plainte, qui dispose de plusieurs occasions de faire valoir son point de vue avant que le Conseil ne décide de former un comité d'enquête ${ }^{42}$ ou de recommander sa destitution $^{43}$, le plaignant ne possède, semble-t-il, à aucune de ces étapes le droit de faire des représentations auprès des différentes instances chargées de traiter sa plainte ${ }^{44}$. Ce n'est qu'en cas de

était fortement répréhensible et qu'elle constituait un manquement à l'honneur et à la dignité de sa charge.

42. Plusieurs dispositions des Procédures vont en ce sens : art. 3.5 c) et 4.1 (demande de commentaires par le président ou le vice-président du Comité sur la conduite des juges), art. 7.2 (lorsque le président ou le vice-président du Comité sur la conduite des juges demande à un avocat de mener une enquête supplémentaire et de rédiger un rapport); art. 9.4 (lorsque le dossier est déféré à un sous-comité); art. 10.3 (étude par le Conseil du rapport d'un sous-comité recommandant la constitution d'un comité d'enquête). En vertu de l'article 64 de la Loi sur les juges, supra note 4, le juge a le droit d'être informé suffisamment à l'avance de l'objet d'une enquête publique à son sujet et doit avoir la possibilité de se faire entendre, de contre-interroger les témoins et de présenter tous les éléments de preuve utiles à sa décharge. L'article 5 (2) du Règlement administratif $\mathrm{du}$ Conseil canadien de la magistrature sur les enquêtes, supra note 33, lui accorde le droit de recevoir, à l'égard des plaintes qu'un comité d'enquête entend examiner, un préavis suffisamment long pour lui permettre d'offrir une réponse complète.

43. L'article $9 \mathrm{du}$ Règlement administratif $d u$ Conseil canadien de la magistrature sur les enquêtes, supra note 33, accorde au juge, dans les trente jours de la réception du rapport du comité d'enquête, le droit de présenter des observations écrites au Conseil et de se présenter en personne devant le Conseil pour y faire une déclaration de vive voix.

44. La jurisprudence a cependant établi que le plaignant a droit à l'équité procédurale, y compris le devoir d'impartialité, à l'étape du traitement de la plainte par le président ou le vice-président du Comité sur la conduite des juges et que le critère applicable est celui de la crainte raisonnable de partialité : Taylor c. Canada (Procureur général), supra note 16, aux pp. 34-39 et 41 (par. 71-85 et 92). 
fermeture 45 et lorsqu'il décide de tenir une enquête publique ${ }^{46}$ que le Conseil est tenu de l'informer de l'évolution du dossier, bien que le directeur exécutif puisse aussi lui faire part d'autres développements ${ }^{47}$. Les Procédures ne lui fournissent aucun accès aux éléments factuels recueillis en rapport avec sa plainte, et ne lui permettent pas même de prendre connaissance des raisons données par le juge pour y répondre, ne serait-ce que pour rectifier les impressions ou les faits qui lui paraîtraient erronés ou incomplets.

Ainsi, le justiciable qui souhaite obtenir la tenue d'une enquête publique à propos de la conduite d'un juge de nomination fédérale doit, par le seul texte de sa plainte: i) convaincre le directeur exécutif que sa plainte est suffisamment sérieuse pour justifier l'ouverture d'un dossier auprès du Conseil canadien de la magistrature; ii) convaincre le président ou le vice-président du Comité sur la conduite des juges que sa plainte est suffisamment sérieuse pour être déférée à un sous-comité, que le repentir éventuel du juge est insuffisant et qu'aucune mesure corrective ne pourrait pallier l'inconduite du juge; iii) convaincre le sous-comité que ses allégations sont suffisamment graves et que l'application d'éventuelles mesures correctives ne peut servir de moyen de disculpation; iv) convaincre le Conseil canadien de la magistrature que les reproches qu'il adresse au juge sont suffisamment graves pour justifier une destitution.

Nombre d'actes considérés comme des inconduites judiciaires n'ont fait l'objet d'aucune enquête publique parce que les instances internes du Conseil ont plutôt décidé de fermer le

45. Art. 3.6, 5.4 et 8.2 (fermeture du dossier par le président ou le viceprésident du Comité sur la conduite des juges), art. 9.8 (fermeture du dossier par un sous-comité), art. 12.1 (fermeture du dossier par l'une ou l'autre des instances du Conseil) des Procédures.

46. Art. 12.6 des Procédures.

47. Art. 12.2 (suspension du dossier pendant l'application de mesures correctives), art. 12.3 (lorsqu'une enquête supplémentaire est demandée à un avocat) et art. 12.4 (transmission du dossier à un sous-comité) des Procédures. 
Les ambiguïtés $d u$

(2008-09) 39 R.D.U.S. processus disciplinaire applicable

aux juges de nomination fédérale

dossier 48 ; à titre d'exemples ${ }^{49}$ : des commentaires discriminatoires ou démontrant une insensibilité à l'égard de groupes sociaux ${ }^{50}$; une condamnation du juge pour conduite avec facultés affaiblies ${ }^{51}$; des propos grossiers dans les échanges du juge avec les avocats 52 ; la discussion en public, par un juge en état d'ébriété, d'affaires dont il était saisis3; l'utilisation du papier à lettre du tribunal pour des affaires personnelles54; des commentaires laissant sous-entendre une présomption de mauvaise foi de la part d'un groupe de juges ${ }^{55}$; une lettre ouverte au premier ministre critiquant un projet de loi ${ }^{56}$; le fait qu'un juge

48. Cette approche du Conseil, mettant fin à des dossiers sans tenir d'enquête même lorsque la plainte d'inconduite est fondée, existe apparemment depuis longtemps. Ainsi, quelques années à peine après la création du Conseil, l'un de ses membres écrivait, à propos de quelques cas où la plainte était fondée, que "la seule démonstration par le Conseil de son intérêt a toujours fait immédiatement rentrer les choses dans l'ordre": Jules Deschênes, "Le Conseil canadien de la magistrature", (1976) 6 R.D.U.S. 375, à la p. 381.

49. À moins de circonstances spéciales, le Conseil a pour principe de ne pas publier le résultat du traitement des plaintes si un comité d'enquête n'est pas établi : Cosgrove, supra note 19, à la p. 741 (par. 68). Fondée sur la volonté légitime de protéger le juge visé par la plainte, cette politique empêche toutefois une appréciation critique de la façon dont les instances internes exercent leur pouvoir. Sauf mention contraire, les quelques décisions mentionnées dans le présent texte proviennent du site internet du Conseil.

50. Décision d'un sous-comité concernant le juge McLung, 19 mai 1999; Décision d'un sous-comité concernant le juge Barakett, 24 juillet 2002; Taylor c. Canada (Procureur général), supra note 16, p. 11 (par. 3).

51. Décision d'un vice-président du comité sur la conduite des juges concernant le juge Alain, 20 août 2007.

52. Décision du président du comité sur la conduite des juges concernant le juge Leask, 20 septembre 2007.

53. Décision rapportée dans le Conseil canadien de la magistrature, Rapport annuel 1990-1991, à la p. 16.

54. Décision rapportée dans le Conseil canadien de la magistrature, Rapport annuel 1996-1997, à la p. 26.

55. Décision rapportée dans le Conseil canadien de la magistrature, Rapport annuel 1999-2000, à la p. 28.

56. Décision d'un sous-comité concernant le juge Angers, 1995, publiée à : (1996) 45 R.D. U.N.-B. 185. 
se soit endormi ou ait donné l'impression de s'endormir au cours d'une audience 57.

Le cadre mis en place par les Procédures n'est sans doute pas étranger au fait que la décision de fermer un dossier, et plus particulièrement celle prise par le président ou le vice-président du Comité sur la conduite des juges, soit parfois contestée en Cour fédérale par le plaignant ${ }^{58}$. Ce phénomène est apparemment propre au Conseil canadien de la magistrature. Compte tenu que la norme de contrôle retenue pour réviser une telle décision est la plus restrictive qui soit59, la possibilité qu'un plaignant réussisse à faire renverser par les tribunaux judiciaires la décision de fermer le dossier relatif à sa plainte est cependant réduite; aucun de ceux qui s'y sont essayés n'a jusqu'à présent réussi à atteindre un tel résultat. À toutes fins pratiques, il en résulte que les diverses instances du Conseil appelées à traiter une plainte exercent leur discrétion avec une autonomie quasiment complète.

Dans les situations où le Conseil canadien de la magistrature constitue un comité d'enquête sans y être tenu par une demande impérative du ministre de la Justice du Canada ou du procureur général d'une province, l'encadrement énoncé aux Procédures produit aussi certains effets à l'égard du juge visé par une plainte. En effet, l'accumulation de preuves, d'analyses et d'appréciations négatives qui se produit au cours du processus de traitement de la plainte constitue l'arrière-plan inévitable d'une enquête publique tenue dans ce contexte. Aucun comité d'enquête ne peut alors être établi sans qu'auparavant, toutes et chacune des diverses instances du Conseil intervenant séparément dans le traitement d'une plainte ne considèrent que les faits reprochés au juge puissent justifier sa destitution. Cette

57. Décision rapportée dans le Conseil canadien de la magistrature, Rapport annuel 2002-2003, à la p. 29.

58. Taylor c. Canada (Procureur général), supra note 16; Crowe c. Juge en chef du Canada, 2007 CF 1209 (C.F. lière inst.), aux par. 32 et 35; Akladyous, supra note 19.

59. Taylor c. Canada (Procureur général), supra note 16, aux pp. 26-29 (par. 46-55); Akladyous, supra note 19, par. 42. 
Les ambiguïtés $d u$

(2008-09) 39 R.D.U.S. processus disciplinaire applicable

superposition d'opinions défavorables au juge ne peut faire autrement que de peser lourdement contre lui au cours de l'enquête publique qui en découle.

Sans remettre en cause ni l'impartialité, ni l'indépendance des comités d'enquête formés par le Conseil canadien de la magistrature, on peut tout de même noter que l'enquête publique tenue dans ces circonstances ne se déroule pas dans un contexte aussi neutre que celui qui entoure l'audition d'un litige devant une cour de justice, où le droit des parties de faire entendre leur cause par un juge ne dépend pas d'une appréciation préalable par le tribunal du mérite des allégations des parties. En raison des caractéristiques structurelles du processus de traitement des plaintes, le comité d'enquête ne peut ignorer que toutes les étapes du processus prévu aux Procédures ont été préalablement franchies de façon défavorable au juge ${ }^{60}$. Ayant déjà fait valoir son point de vue à plusieurs reprises auprès des diverses instances du Conseil sans avoir réussi à les convaincre du bien-fondé de sa position, le juge dont la conduite fait l'objet d'une enquête publique se trouve dans une position vulnérable. Il ne peut vraisemblablement sortir complètement exonéré d'une telle enquête; tout au plus peut-il espérer éviter une recommandation de destitution.

Enfin, la structure contenue aux Procédures entraîne aussi certaines conséquences pour le moins discutables quant à l'exercice de la discrétion attribuée au Conseil par la Loi sur les juges. Dans le cadre mis en place par les Procédures, chacune des instances intervenant successivement dans le processus de traitement des plaintes avant la constitution d'un comité d'enquête possède un pouvoir décisionnel définitif quant à la

60. Le Conseil canadien de la magistrature a déjà déclaré qu'il avait "pour politique de ne pas s'engager à l'aveuglette" dans des enquêtes publiques, "sans trop savoir ce qui en ressortira", et qu'il ne tient de telles enquêtes "que dans les affaires où il existe, prima facie, un motif de révocation": Conseil canadien de la magistrature, Rapport annuel 1990-1991, à la p. 11; dans le même sens, voir aussi: Conseil canadien de la magistrature, Rapport annuel 1991-1992, à la p. 10. 
Les ambigütés $d u$

processus disciplinaire applicable (2008-09) 39 R.D.U.S. aux juges de nomination fédérale

fermeture du dossier61; ce n'est que si, dans son entière discrétion, elle considère la plainte suffisamment sérieuse que celle-ci est déférée à une autre instance pour examen. Il n'existe aucun mécanisme d'appel ou de révision auprès du Conseil siégeant en plénière en cas de fermeture du dossier à un stade préliminaire.

Cet enchaînement particulier des responsabilités respectives des diverses instances internes du Conseil aboutit au paradoxe suivant : un plus grand poids est accordé à l'opinion du président ou du vice-président du Comité sur la conduite des juges, quant au sérieux de la plainte, qu'à celle d'un sous-comité ou du Conseil canadien de la magistrature agissant en plénière ${ }^{62}$. La voix d'un seul membre du Conseil prime celle d'un sous-groupe ainsi que celle de l'ensemble des membres. Sans doute est-il peu pratique, et probablement inutile, que le Conseil canadien de la magistrature se prononce en plénière à propos de chacune des plaintes qu'il reçoit. Une grande partie des plaintes consistent apparemment en une appréciation du bien-fondé du jugement rendu par le juge visé par la plainte, une fonction qui incombe aux

61. La Cour d'appel fédérale a cependant précisé que le processus d'enquête auprès du Conseil est continu et que la décision de l'une de ses instances de classer une affaire peut toujours être revue sur la base de nouvelles informations : Taylor c. Canada (Procureur général), supra note 16, aux pp. 41-42 (par. 94). Il semble que les demandes de réexamen ne soient pas exceptionnelles: Conseil de la magistrature, Rapport annuel 20072008, à la p. 15.

62. Il dispose d'ailleurs d'un vaste pouvoir d'appréciation dans la manière d'examiner une plainte et n'est pas tenu d'entendre des témoins : Taylor c. Canada (Procureur général), supra note 16, p. $44-45$ (par. 106-107). Il semble aussi particulièrement problématique que le président ou le viceprésident du Comité sur la conduite des juges prenne sa décision en tentant d'anticiper ce que déciderait un sous-comité s'il lui déférait la plainte. Ainsi, dans la Décision du président du comité sur la Conduite des juges concernant le juge Bastarache, 15 mars 2001, le président du Comité sur la conduite des juges exprime son avis qu'aucun sous-comité ne pourrait conclure que les commentaires du juge visé par la plainte sapent la confiance du public en son impartialité. S'il lui en avait donné l'occasion, un sous-comité aurait bien pu prendre une autre position. 
Les ambiguïtés $d u$

(2008-09) 39 R.D.U.S. processus disciplinaire applicable

tribunaux d'appel et non au Conseil63. Un processus préliminaire permettant de départager les plaintes, afin d'identifier celles qui concernent des manquements déontologiques, et de recueillir un minimum d'informations pertinentes paraît ainsi indispensable.

Toutefois, le Conseil devrait se réserver la décision finale quant à la fermeture d'un dossier lorsque la plainte fait état d'une véritable inconduite judiciaire, quel qu'en soit par ailleurs le degré de gravité64. C'est au Conseil que la Loi sur les juges confie le soin d'enquêter à propos des plaintes et la seule autre instance qu'elle identifie spécifiquement à cette fin est le comité d'enquête. Il semblerait beaucoup plus conforme à l'esprit de la loi que la volonté de fermer un dossier exprimée par les instances internes du Conseil prenne la forme d'une recommandation adressée à celui-ci, pour qu'il en décide en dernier ressort. C'est d'ailleurs dans ce cadre que le Conseil reçoit les rapports des comités d'enquête quant à l'opportunité d'une recommandation de destitution $^{65}$. La situation actuelle peut donner l'impression que, pour le Conseil, la décision de fermer un dossier de plainte sans tenir d'enquête publique ne présente pas autant d'importance, au plan juridique, que celle de tenir une enquête publique à propos de cette plainte.

En outre, cette survalorisation de l'opinion des instances saisies de la plainte avant l'étape du comité d'enquête produit un curieux résultat en ce qui a trait à la profondeur de l'analyse des plaintes par le Conseil. Selon les principes établis, les comités d'enquête recueillent une preuve qui permet au Conseil, en fonction de sa propre appréciation de la situation, de faire ses recommandations au ministre de la Justice quant à la destitution

63. Le Conseil le rappelle d'ailleurs dans tous ses rapports annuels. Cette distinction a aussi été évoquée dans le Rapport Cosgrove (C.E.), aux par. 121-126.

64. Un auteur a d'ailleurs critiqué le système de filtrage définitif des plaintes par un seul juge: H. Patrick Glenn, "Indépendance et déontologie judiciaires", (1995) 55 R. du B. 295, à la p. 309.

65. Art. 65 de la Loi sur les juges, supra note 4, et art. 8 à 13 du Règlement administratif du Conseil de la magistrature sur les enquêtes, supra note 33. 
Les ambiguïtés $d u$

processus disciplinaire applicable (2008-09) 39 R.D.U.S. aux juges de nomination fédérale

du juge66. Leurs rapports ne jouissent en effet d'aucune autorité particulière auprès du Conseil et ce dernier est entièrement libre de réviser leurs conclusions ${ }^{67}$. À l'inverse, le président ou le viceprésident du Comité sur la conduite des juges, ainsi que le souscomité à qui une plainte est déférée, peuvent imposer au Conseil leur décision de fermer un dossier, bien que leur enquête soit moins approfondie que celle menée par un comité d'enquête68. En dépit du fait qu'il remplisse la fonction d'enquête la plus élaborée, le comité d'enquête est ainsi la seule instance dont les conclusions ne peuvent, en aucun cas, s’imposer au Conseil.

\section{La sanction de l'inconduite judiciaire}

Bien que la Loi sur les juges soit rédigée de façon telle que le pouvoir d'enquête du Conseil canadien de la magistrature couvre un large éventail de plaintes ${ }^{69}$, elle ne précise pas les actes

66. C'est la position prise par le comité d'enquête dans le Rapport Matlow (C.E.), aux par. 12 et 13 : "Le Comité d'enquête constitue en réalité le moyen par lequel le [Conseil canadien de la magistrature] effectue l'enquête et recueille les renseignements factuels nécessaires pour tirer des conclusions et formuler des recommandations au Ministre"; "Il incombe donc au Comité d'enquête d'indiquer tous les résultats nécessaires pour que le [Conseil canadien de la magistrature] formule la recommandation qu'il juge appropriée, indépendamment des conclusions ou de la recommandation du Comité d'enquête et de ce que le Comité d'enquête considère comme un fondement factuel suffisant pour lui permettre de formuler une recommandation". Le Conseil a adopté un point de vue similaire, en précisant qu'il ne siégeait pas en appel des rapports des comités d'enquête : Rapport Matlow (C.C.M.), aux par. 52-55 et 67.

67. Ce pouvoir du Conseil de réviser les recommandations d'un comité d'enquête est bien réel, tel que le démontre la réception qu'il a réservée au Rapport Berger (C.E.), dont la conclusion que la plainte était bien fondée a été désavouée par le Conseil, au Rapport Boilard (C.E.), dont le raisonnement a été désavoué par le Conseil, et au Rapport Matlow (C.E.), dont la recommandation de destitution a été écartée par le Conseil.

68. Dans le Rapport Berger (C.E.), le comité d'enquête faisait fort justement remarquer que "the full facts of a case may not be known or understood until an investigation has taken place" (p. 385).

69. Le paragraphe 63 (2) de la Loi sur les juges, supra note 4, se lit ainsi : "Le Conseil peut en outre enquêter sur toute plainte ou accusation relative à un juge d'une juridiction supérieure". Il semble donc plus large que le paragraphe 63 (1), qui prévoit que les enquêtes menées par le Conseil à 
Les ambiguïtés $d u$

(2008-09) 39 R.D.U.S. processus disciplinaire applicable

d'inconduite judiciaire pouvant y donner ouverture. Par ailleurs, aucune sanction n'est autorisée contre un juge par cette loi, quel que soit le degré de gravité de l'inconduite constatée par le Conseil dans le cadre de son analyse du dossier. Par divers procédés, le Conseil a tenté de pallier ces lacunes afin, de toute évidence, de préserver l'efficacité du mécanisme de plainte et de rendre les juges de nomination fédérale véritablement redevables de leur conduite. Ces procédés, cependant, n’apparaissent pas entièrement satisfaisants.

\subsection{L'assise des obligations déontologiques}

Pour en arriver à une recommandation de destitution, le Conseil canadien de la magistrature doit être d'avis que le juge est inapte à remplir utilement ses fonctions en raison de l'âge, d'une invalidité, d'un manquement à l'honneur et à la dignité, d'un manquement aux devoirs de sa charge, ou encore en raison d'une situation d'incompatibilité, qu'elle soit imputable au juge ou à toute autre cause ${ }^{70}$. Étant donné que les dispositions législatives et constitutionnelles ${ }^{71}$ consacrant l'inamovibilité des juges de nomination fédérale ne mentionnent aucun critère autre que la "bonne conduite», cette énumération représente une rare indication des motifs que le Parlement fédéral et ses diverses composantes - dont le concours est nécessaire à une éventuelle destitution - considèrent pertinents pour en arriver à une telle décision ${ }^{72}$.

la demande du ministre de la Justice du Canada ou des procureurs généraux des provinces portent sur "les cas de révocation au sein d'une juridiction supérieure pour tout motif énoncé aux alinéas 65 (2) a) à d)".

70. Art. 65 (2) de la Loi sur les juges, supra note 4 . La Cour fédérale a décidé que l'invalidité doit être permanente pour justifier la destitution : Gratton, supra note 34 , aux pp. 792-796.

71. Voir supra note 9.

72. La Cour fédérale a exprimé l'avis que les circonstances dans lesquelles le Conseil peut enquêter à propos de la conduite d'un juge sont nécessairement limitées à celles qui permettent sa destitution par le gouverneur général : Gratton, supra note 34, à la p. 781 . 
Les dispositions de la Loi sur les juges qui attribuent au Conseil canadien de la magistrature la responsabilité de traiter les plaintes formulées à l'égard des juges n'imposent toutefois aucune obligation déontologique à ces derniers. Elles ne font que préciser les circonstances dans lesquelles le Conseil peut recommander la destitution au ministre de la Justice du Canda. Pourtant, les concepts de "manquement à l'honneur et à la dignité", de "manquement aux devoirs de sa charge" et de "situation d'incompatibilité" supposent nécessairement l'existence de normes de conduite qui s'imposent aux juges de nomination fédérale, mais sans en indiquer la source ni en préciser les contours. Cette technique de rédaction législative met donc l'emphase sur l'aboutissement du processus de plainte: une sanction est identifiée - une éventuelle destitution - sans que les obligations déontologiques dont elle vise à assurer l'observation n'aient été préalablement définies.

Jusqu'à présent, on peut constater que les comités d'enquête n'ont pas vraiment cherché à préciser le sens des termes utilisés par la Loi sur les juges pour identifier les motifs justifiant une recommandation de destitution, ni même à définir l'inconduite judiciaire ${ }^{73}$. Ils puisent les obligations déontologiques des juges de nomination fédérale à diverses sources: un consensus au sein des pays de common $l a w^{74}$, les principes déontologiques développés à l'égard des juges de nomination provinciale $^{75}$ ou encore une déclaration de principes internationale ${ }^{76}$, à titre d'exemples. Leur analyse de la conduite du juge procède de façon très concrète, au cas par cas, avant qu'ils la qualifient à la lumière des concepts utilisés par la Loi sur les juges.

73. L'exposé le plus élaboré à ce sujet se trouve dans le Rapport Matlow (C.E.), aux par. 119-146.

74. Rapport Berger (C.E.), à la p. 385.

75. Rapport Flynn (C.E.), aux par. 46-49; Rapport Matlow (C.E.), au par. 123.

76. Rapport Flynn (C.E.), au par. 47. 
Les ambiguïtés $d u$

(2008-09) 39 R.D.U.S. processus disciplinaire applicable

aux juges de nomination fédérale

Officiellement suggérée au Conseil canadien de la magistrature au milieu des années 199077, l'adoption d'un code de déontologie destiné aux juges de nomination fédérale aurait permis de combler cette lacune, en apportant à tout le moins un premier niveau de précision quant aux devoirs des membres de la magistrature. Cette solution a cependant été écartée par le Conseil. Il est vrai que la Loi sur les juges ne lui attribue aucun pouvoir en ce sens ${ }^{78}$. Cependant, quelques déclarations du Conseil ${ }^{79}$ laissent croire que l'absence d'habilitation législative ne constitue pas l'unique motif de son refus: le Conseil semble considérer plus conforme à l'indépendance judiciaire l'élaboration d'un simple guide de conduite non contraignant, comme les Principes de déontologie judiciaire ${ }^{80}$ qu'il a publiés en 1998 à l'intention des juges assujettis à sa compétence d'enquête.

Cette perspective semble fondée sur la prémisse qu'aucune règle déontologique ne pourrait être imposée à un juge par la contrainte sans porter atteinte à son indépendance. Elle adopte une conception quasi facultative de la déontologie judiciaire, la bonne volonté du juge en constituant le moteur principal et sa

77. Martin Lawrence Friedland, Une place à part: l'indépendance et la responsabilité de la magistrature au Canada, Ottawa, Conseil canadien de la magistrature, 1995, aux pp. 170-174.

78. Le Conseil l'a d'ailleurs rappelé à quelques reprises : Conseil canadien de la magistrature, Rapport annuel 1996-1997, à la p. 35; Conseil canadien de la magistrature, Rapport annuel 1997-1998, à la p. 31.

79. "Le professeur Friedland avait proposé la préparation d'un code de conduite, mais le groupe de travail du Conseil en est finalement venu à la conclusion qu'il était plus approprié de définir et d'énoncer des principes déontologiques qui pourraient guider les juges dans leur conduite et que cette façon de procéder était plus conforme à l'indépendance judiciaire que l'adoption d'un code de conduite en bonne et due forme" : Conseil canadien de la magistrature, Rapport annuel 1996-1997, à la p. 34; à propos d'un ouvrage antérieur du Conseil qui évitait d'établir des règles de conduite obligatoires, le Conseil écrivait aussi que "procéder ainsi aurait pu porter atteinte au principe de l'indépendance judiciaire, qui laisse les juges seuls responsables de leur conduite": Conseil canadien de la magistrature, Rapport annuel 1990-1991, à la p. 10.

80. Conseil canadien de la magistrature, Principes de déontologie judiciaire, 1998. 
probité, la seule garantie de réalisation ${ }^{81}$. Pourtant, on transformerait l'indépendance judiciaire en une singulière sphère d'impunité, cadrant mal avec la primauté du droit, en utilisant ce principe constitutionnel de première importance pour placer les juges à l'abri des contraintes nécessaires à l'exercice des fonctions publiques dont ils sont investis. Il semble difficile de voir comment un juge pourrait légitimement invoquer l'indépendance judiciaire pour s'opposer, par exemple, à l'obligation qui lui serait faite par un code de déontologie de préserver son impartialité, de ne pas porter atteinte aux valeurs fondamentales inscrites dans la Constitution, de rendre ses jugements en fonction des règles de droit plutôt que selon ses préférences personnelles, ou encore de s'abstenir d'activités politiques ${ }^{82}$. La conduite des juges de nomination fédérale ne peut échapper à toute norme dès lors qu'elle ne présente pas le degré de gravité suffisant pour justifier une destitution.

Depuis la publication des Principes de déontologie judiciaire, des rapports d'enquête remis au Conseil y ont fait référence pour identifier les obligations déontologiques des juges de nomination fédérale ${ }^{83}$. Dans l'une de ses recommandations au ministre de la

81. Dans Ruffo c. Conseil de la magistrature, supra note 7, p. 332 (par. 110), la Cour suprême du Canada a accrédité cette conception de la déontologie judiciaire : "La règle de déontologie, en effet, se veut une ouverture vers la perfection. Elle est un appel à mieux faire, non par la sujétion à des sanctions diverses mais par l'observation de contraintes personnellement imposées"; cette position a parfois été reprise par les instances du Conseil : Rapport Matlow (C.E.), au par. 124; Rapport Matlow (C.C.M.), au par. 95.

82. Le Code de déontologie de la magistrature, R.R.Q. c. T-16, r. 4.1, imposant des obligations déontologiques aux juges de nomination provinciale au Québec, a été considéré valide par les tribunaux : Ruffo c. Le Conseil de la magistrature, [1989] R.J.Q. 2432 (C.S.), p. 2444-2451; confirmé par la Cour d'appel dans Ruffo c. Conseil de la magistrature du Guébec, EYB 1992-63960, par. 21 à 26; désistement de pourvoi à la Cour suprême du Canada : [1995] 1 R.C.S. x. Contrairement à ce qu'écrit le Conseil dans le Rapport Matlow (C.C.M.), par. 94, il y a bel et bien deux modèles d'encadrement de la déontologie judiciaire au Canada, l'un qui impose des obligations aux juges et l'autre qui leur prodigue des conseils.

83. Rapport Flynn (C.E.), aux par. 50-54 et 57; Rapport Matlow (C.E.), aux par. 123-147. 
Les ambiguïtés $d u$

(2008-09) 39 R.D.U.S. processus disciplinaire applicable

Justice ${ }^{84}$, soit à l'étape ultime de la compétence d'enquête qui lui est confiée par la Loi sur les juges, le Conseil canadien de la magistrature a cependant réaffirmé qu'ils n'énoncent pas de règles impératives, bien que ses instances internes puissent s'en inspirer dans le traitement des plaintes. Quoiqu'elle soit conforme à la perspective dans laquelle ces Principes ont été élaborés, cette position laisse néanmoins en plan des questions fondamentales 85 : les juges de nomination fédérale ont-ils une obligation légale de respecter certaines normes déontologiques en rapport avec la fonction qu'ils exercent; dans l'affirmative, quel est le fondement juridique de cette obligation et comment peut-on identifier les normes déontologiques qui en découlent?

Deux conséquences importantes découlent de l'absence de normes de conduite explicites pour les juges de nomination fédérale, en ce qui concerne le traitement des plaintes dirigées contre eux en vertu de la Loi sur les juges. La première est qu'il incombe aux instances chargées de traiter ces plaintes d'identifier de manière empirique ce qu'elles considèrent comme des manquements déontologiques parmi les circonstances portées à leur attention. De cette façon, les obligations déontologiques des juges ne sont définies qu'après la survenance des faits générateurs de la plainte. Sans indication ferme quant aux limites acceptables de sa conduite, le juge visé par une plainte court ainsi le risque d'apprendre de l'une ou l'autre de ces instances qu'il a fait défaut de respecter des obligations qui ne lui avaient pas été auparavant détaillées. Cette situation problématique a d'ailleurs déjà conduit un comité d'enquête à invoquer l'absence d'une norme de conduite préalable comme un motif suffisant pour refuser de recommander la destitution d'un juge, alors qu'il avait pourtant conclu que sa conduite aurait justifié une telle recommandation 86 .

84. Rapport Matlow (C.C.M.), aux par. 94 et 99.

85. À ce sujet, voir Huppé, supra note 7, aux pp. 101-106.

86. "We do not, however, think it would be fair to set standards ex post facto to support a recommendation for removal in this case": Rapport Berger (C.E.), à la p. 392. Dans le même sens, en refusant d'entériner la recommandation de destitution formulée par un comité d'enquête, le Conseil canadien de la magistrature écrivait ce qui suit dans le Rapport 
Les ambiguïtés $d u$

processus disciplinaire applicable (2008-09) 39 R.D.U.S. aux juges de nomination fédérale

Il survient aussi parfois des débats au sein du Conseil quant à l'existence même d'une obligation déontologique, alors que le processus de traitement des plaintes devrait plutôt servir à vérifier la présence d'une contravention à des normes connues et préétablies. Ainsi, une lecture comparée des motifs majoritaires et des motifs minoritaires du Rapport Marshall (C.E.) montre un désaccord entre les membres du comité d'enquête relativement à l'existence d'une obligation déontologique quant à la formulation de commentaires dans un jugement à propos de la conduite d'une partie au litige ${ }^{87}$. De même, les différents points de vue exprimés dans le traitement des plaintes déposées à l'égard des juges Boilard $^{88}$ et Matlow ${ }^{89}$ permettent difficilement de savoir s'il existe des contraintes déontologiques relativement à la récusation d'un juge dans un dossier dont il est saisi, et quelles seraient ces contraintes. Les juges visés par une plainte font les frais de telles incertitudes normatives, quel que soit le résultat du processus engagé devant le Conseil.

En raison de la fragmentation du pouvoir décisionnel entre les diverses instances internes du Conseil canadien de la magistrature ${ }^{90}$, une seconde conséquence découle de l'absence de normes de conduite explicites : le risque d'un manque d'uniformité dans la mise en ouvre de la déontologie judiciaire. Sans code de déontologie formel pour encadrer son analyse, chaque président ou vice-président du Comité sur la conduite des juges, chaque sous-comité formé pour examiner une plainte, met en application sa propre conception des obligations déontologiques des juges. Puisque le Conseil canadien de la magistrature ne révise pas leurs

Matlow (C.C.M.) : "There are limits to what a judge can do in pursuit of his or her personal or private interests and this case serves as notice to all judges of the limitations that properly apply to a judge's right to pursue civic or personal interests while serving as a judge" (par. 185).

87. Rapport Marshall (C.E.), à la p. 37 (motifs majoritaires) et à la p. 24 (motifs minoritaires).

88. Rapport Boilard (C.E.), au par 98; Rapport Boilard (C.C.M.).

89. Rapport Matlow (C.E.), aux par. 85-95, 136-146, 170-173 et 181-187; Rapport Matlow (C.C.M.), aux par. 124-144 (motifs majoritaires) et 43-77 motifs minoritaires).

90. Voir supra la section 1.2. 
Les ambiguïtés $d u$

(2008-09) 39 R.D.U.S. processus disciplinaire applicable

aux juges de nomination fédérale

décisions lorsque le dossier est fermé sans la tenue d'une enquête publique, il est privé par les Procédures qu'il a lui-même adoptées de la possibilité d'unifier leur évaluation des cas concrets soulevés par ces plaintes. Il ne peut garantir aucune uniformité dans la façon dont ses instances internes définissent et apprécient l'inconduite judicaire, sauf à l'égard du nombre infime de plaintes qui atteignent le stade de l'examen par un comité d'enquête.

Dans le Rapport Matlow (C.C.M.), le Conseil canadien de la magistrature a fermement exprimé la position que les comités d'enquête ne se prononcent pas de façon définitive quant à l'appréciation de ce qui constitue une inconduite judiciaire et qu'il lui incombe d'établir des principes généraux à cet égard ${ }^{91}$. Cette règle devrait recevoir application non seulement lorsque les instances internes du Conseil sont d'avis qu'une enquête publique à propos de la conduite d'un juge est nécessaire, mais aussi lorsqu'elles sont d'avis que son inconduite ne justifie pas une telle enquête. Autrement, pour la quasi-totalité des plaintes, l'inconduite judiciaire est ce que les instances internes du Conseil en pensent.

\subsection{Les formes de l'intervention disciplinaire}

Une constatation d'inconduite ne représente que la première partie de la mission du Conseil canadien de la magistrature; il lui faut ensuite déterminer si cette inconduite atteint un degré suffisant pour justifier une recommandation de destitution $^{92}$. À cette fin, les comités d'enquête ont adopté un critère très exigeant ${ }^{93}$, entériné par les tribunaux supérieurs en ce

91. "Instead, given the need to ensure uniformity and therefore fair and equal treatment, it is the CJC, and not the individual inquiry committees, that should bring its own independent judgment to bear and ultimately confirm the general principles as to the scope of sanctionable conduct, : Rapport Matlow (C.C.M.), au par. 56.

92. Le Conseil a confirmé que l'appréciation d'une plainte comporte ces deux étapes : Rapport Matlow (C.C.M.), au par. 166; Rapport Cosgrove (C.C.M.), au par. 15.

93. Ce critère consiste à déterminer si la conduite reprochée au juge porte "si manifestement et si totalement atteinte à l'impartialité, à l'intégrité et à 
qui a trait tant à la destitution des juges de nomination fédérale ${ }^{94}$ qu'à celle des juges de nomination provinciale ${ }^{95}$. Le premier volet de ce critère est consacré à une appréciation de la conduite du juge par rapport aux principes fondamentaux qui encadrent l'exercice de la fonction judiciaire : l'impartialité, l'indépendance et l'intégrité. Le second volet évalue les effets de la conduite du juge à l'égard de la confiance que les institutions judiciaires doivent inspirer à la population et qui fonde leur légitimité dans la société.

Pour appliquer ce critère, des comités d'enquête ${ }^{96}$ ont eu recours à une fiction largement utilisée en droit canadien : quelle perception une personne raisonnable et bien informée aurait-elle de la conduite du juge? Cette méthode renvoie, en définitive, à la conception que les membres des comités d'enquête se font de ce justiciable idéal dont ils cherchent à deviner l'opinion, puisque la perception réelle du public à l'égard de la conduite du juge leur est inconnue ${ }^{97}$. Les caractéristiques objectives de cette personne

l'indépendance de la magistrature qu'elle ébranle la confiance du justiciable ou du public en son système de justice et rend le juge incapable de s'acquitter des fonctions de sa charge". Formulé dans le Rapport Marshall (C.E.), à la p. 28, il a été repris de façon constante par la suite, malgré certaines variantes de forme dans son libellé : Rapport Bienvenue (C.E.), à la p. 42; Rapport Flynn (C.E.), au par. 35; Rapport Boilard (C.E.), à la p. 49 (par. 112); Rapport Matlow (C.E.), par. 111-112 et Rapport Matlow (C.C.M.), aux par. 96-97 et 164; Rapport Cosgrove (C.E.), au par. 131; Rapport Cosgrove (C.C.M.), au par. 19.

94. Taylor c. Canada (Procureur général), supra note 16, aux pp. 31-32 (par. 63).

95. Re Therrien, supra note 20, à la p. 96 (par. 147); Moreau-Bérubé c. Nouveau-Brunswick (Conseil de la magistrature), [2002] 1 R.C.S. 249, à la p. 281 (par. 51).

96. Rapport Bienvenue (C.E.), aux pp. 60-63, Rapport Flynn (C.E.), au par. 36.

97. Dans le Rapport Bienvenue (C.E.), le comité d'enquête a ainsi considéré irrecevable un sondage d'opinion auprès de la population, en déclarant qu'il ne serait pas sage "de prendre en compte des sondages d'opinion pour juger de la perception du public à l'égard de la conduite d'un juge ou à l'égard de la confiance dont jouit l'appareil judiciaire" (p. 59); dans le rapport par lequel le Conseil a entériné ce rapport d'enquête, les membres dissidents du Conseil ont exprimé leur désaccord avec ce point de vue : Rapport Bienvenue (C.C.M.), à la p. 85. 
Les ambiguïtés $d u$

(2008-09) 39 R.D.U.S. processus disciplinaire applicable

raisonnable n'ont d'ailleurs pas été identifiées ${ }^{98}$, notamment en ce qui a trait à l'ampleur de ses connaissances juridiques ou à sa familiarité avec les conditions dans lesquelles la fonction judiciaire est exercée.

Surtout, on n'a pas précisé en quoi cette personne raisonnable et bien informée se distingue des membres du comité d'enquête. Serait-il possible, même d'un point de vue purement théorique, que la perception des membres d'un comité d'enquête puisse substantiellement différer de celle d'une personne raisonnable? Dans la mesure où il n'existe aucune différence appréciable entre l'analyse effectuée par les membres du comité d'enquête et celle théoriquement faite par une personne raisonnable, le recours à une telle abstraction ajoute une complication inutile au critère utilisé pour déterminer l'opportunité d'une recommandation de destitution. Les comités d'enquête ou le Conseil pourraient plutôt définir eux-mêmes les conditions objectives permettant de maintenir la confiance du public dans ses institutions judiciaires.

Bien qu'une plainte puisse faire état de toute inconduite de la part d'un juge99, la Loi sur les juges ne traite pas de la sanction des inconduites mineures, insuffisamment graves pour justifier une recommandation de destitution. Elle n'autorise pas même le Conseil canadien de la magistrature à prononcer un blâme ou une réprimande à l'égard d'un juge, ni à lui imposer d'autres sanctions

98. Le membre dissident du comité d'enquête ayant remis le Rapport Bienvenue (C.E.) soulignait à juste titre les difficultés de ce critère, en s'interrogeant à propos de l'identité du groupe social dans lequel se situe cette personne raisonnable (p. $28 \mathrm{du}$ rapport dissident).

99. "Un comité d'enquête déclarait ainsi que [...] le paragraphe 63 (2) s'étend aux plaintes concernant toute inconduite, allant des fautes les plus mineures jusqu'aux écarts de conduite les plus graves" : Dans l'affaire de l'enquête du Conseil canadien de la magistrature concernant le juge Paul Cosgrove (C.E.), 16 décembre 2004, à la p. 8 (par. 10). L'article 5 (1) du Règlement administratif du Conseil canadien de la magistrature sur les enquêtes, supra note 33, autorise un comité d'enquête à examiner toute plainte ou accusation pertinente formulée contre un juge et qui est portée à son attention. 
disciplinaires ${ }^{100}$; celui-ci l'a d'ailleurs fait remarquer à plusieurs reprises dans ses rapports annuels ${ }^{101}$. La seule recommandation qui lui est permise par la Loi sur les juges concerne l'opportunité de destituer le juge.

Les rapports d'enquêtes fournissent ainsi plusieurs exemples de conduites décriées par des comités d'enquête, mais qui ne donnent lieu à aucune sanction contre le juge : l'utilisation des services d'une entreprise offrant des spectacles de striptease en privé102; le retard continuel à rendre jugement ${ }^{103}$; la participation à un débat politique ${ }^{104}$; le blâme exagérément sévère adressé à la victime d'une erreur judiciaire105; une récusation décidée par le juge sans motifs valables ${ }^{106}$. Un comité d'enquête a même considéré que le fait de ne pas dénoncer formellement une inconduite judiciaire qu'il réprouvait, sans la juger suffisante pour justifier la destitution, aurait pu porter atteinte à la confiance de la population dans les institutions judiciaires ${ }^{107}$.

À cet égard, le Conseil canadien de la magistrature n'a pas jugé approprié de préciser le fondement juridique des conclusions

100. Dans le Rapport Berger (C.E.), aux pp. 384-385, le comité d'enquête a refusé de déterminer si le Conseil canadien de la magistrature possède le pouvoir de réprimander le juge ou d'imposer une sanction moindre qu'une recommandation de destitution.

101. À titre d'exemple, voir: Conseil canadien de la magistrature, Rapport annuel 1989-1990, à la p. 7; Conseil canadien de la magistrature, Rapport annuel 1990-1991, à la p. 15; Conseil canadien de la magistrature, Rapport annuel 1993-1994, à la p. 13. Un comité d'enquête a néanmoins évoqué cette sanction, mais sans la retenir : Rapport Cosgrove (C.E.), aux par. 188 et 200.

102. Rapport d'enquête relaté dans le Rapport annuel du Conseil canadien de la magistrature, 1996-1997, à la p. 19.

103. Rapport d'enquête relaté dans le Rapport annuel du Conseil canadien de la magistrature, 1996-1997, aux pp. 19-20.

104. Rapport Berger (C.E.), à la p. 391; Rapport Flynn (C.E.), aux par. 71-76.

105. Rapport Marshall (C.E.), à la p. 37.

106. Rapport Boilard (C.E.), à la p. 50 (par. 114). L'appréciation du comité d'enquête dans ce dossier a cependant été désapprouvée par le Conseil dans son rapport au ministre de la Justice du Canada : Rapport Boilard (C.C.M.).

107. Rapport Marshall (C.E.), à la p. 37. 
Les ambiguïtés $d u$

(2008-09) 39 R.D.U.S. processus disciplinaire applicable

exceptionnelles qu'il a prononcées dans le Rapport Matlow (C.C.M.)108 : désapprouvant expressément sa conduite, il a obligé le juge à présenter des excuses écrites dans une forme acceptable au Conseil, il lui a ordonné d'assister à un séminaire portant sur la déontologie judiciaire et lui a aussi interdit de participer à tout débat public sans obtenir au préalable une opinion favorable du Comité consultatif sur la déontologie judiciaire109. Les dix-sept membres concourant à l'opinion majoritaire n'ont mentionné au soutien de leurs conclusions aucune disposition législative, ni principe de droit, qui donnerait une telle compétence au Conseil canadien de la magistrature.

Quelques dispositions des Procédures offrent aux diverses instances internes du Conseil la faculté d'exposer au juge visé par la plainte une évaluation de sa conduite et de lui exprimer leurs préoccupations. Une première occasion est fournie au président ou au vice-président du Comité sur la conduite des juges au moment où il ferme un dossier parce qu'il ne considère pas nécessaire de prendre d'autres mesures et que le juge reconnaît que sa conduite était déplacée110; cette possibilité est également offerte au sous-comité ou au Conseil lorsqu'ils ferment un dossier ${ }^{111}$. Aucune disposition de la Loi sur les juges n'autorise

108. Rapport Matlow (C.C.M.), au par. 185-186.

109. Ce comité donne des avis confidentiels aux juges qui le consultent à propos de questions déontologiques; il a émis une soixantaine d'opinions durant ses cinq premières années d'existence: Georgina R. Jackson, "The Mystery of Judicial Ethics : Deciphering the "Code", (2005) 68 Sask. L. Rev. 1, aux pp. 7-8.

110. Art. 5.2 des Procédures. La Cour d'appel fédérale a fait référence à un tel pouvoir sans se prononcer sur sa légalité, en précisant qu'il ne peut être exercé que lorsque le juge reconnaît le caractère déplacé ou répréhensible de sa conduite et en indiquant que la conduite du juge doit présenter une certaine gravité pour qu'il puisse être exercé : Taylor c. Canada (Procureur général), supra note 16, aux pp. 31, 33-34 et 46 (par. 61-62, 69 et 111$)$.

111. Art. 9.7 et 10.5 des Procédures; à l'une ou l'autre de ces étapes, il n'est cependant pas nécessaire que le juge visé par la plainte reconnaisse le caractère déplacé de sa conduite pour que ces préoccupations puissent lui être exprimées. Le règlement administratif remplacé par ces Procédures autorisait plutôt les instances du Conseil, dans les mêmes 
cependant une telle intervention auprès d'un juge visé par une plainte $^{112}$.

Tout comme la dénonciation publique de la conduite d'un juge par un comité d'enquête ou par le Conseil, cette solution ne peut être envisagée comme une panacée aux insuffisances de la Loi sur les juges. Elle pourrait même parfois être considérée, précisément en raison de l'absence d'une assise législative qui l'autoriserait, comme une pression indue exercée à son égard. Le président ou le vice-président du Comité sur la conduite des juges, par exemple, n'exerce aucune autorité à l'égard du juge visé par une plainte, ni au plan judiciaire, ni au plan administratif 113 . L'évaluation qu'il fait de la conduite du juge, de même que les préoccupations dont il lui fait part, ne constituent que l'expression de son opinion personnelle. Afin de protéger l'indépendance judiciaire, il semble indispensable que tout mécanisme permettant d'adresser directement une critique à un juge, en privé ou en public, soit encadré par des règles de droit qui précisent les circonstances permettant une telle intervention et la portée qu'elle peut prendre.

Ainsi, il est arrivé qu'un juge réagisse fortement à la désapprobation que manifestait un sous-comité à l'égard de sa conduite ${ }^{114}$, en se dessaisissant d'un procès qu'il présidait depuis

circonstances, à exprimer leur désapprobation quant à la conduite du juge : Règlement administratif du Conseil, supra note 24, art. 50 (2) et 55 (2).

112. Un auteur soutient que cette pratique trouve son fondement dans les objets généraux du Conseil et dans l'intérêt public: Ed Ratushny, "Speaking As Judges : How Far Can They Go?», (1999-2000) 11 R.N.D.C. 293, à la p. 311; un tel fondement paraît cependant largement insuffisant pour justifier des interventions aussi directes auprès d'un juge.

113. L'article 3.2 des Procédures empêche d'ailleurs le président et les viceprésidents du Comité sur la conduite des juges d'être saisis d'une plainte concernant un juge faisant partie du même tribunal qu'eux. En vertu des articles 9.1 b) et 10.2 , il en est de même des membres d'un souscomité et des membres d'un comité d'enquête.

114. Incident rapporté dans le Rapport Boilard (C.E.), aux pp. 12-15. Tel que l'indique la Décision d'un sous-comité concernant le juge Boilard, 15 juillet 
Les ambiguïtés $d u$

(2008-09) 39 R.D.U.S. processus disciplinaire applicable

plusieurs mois puisqu'il considérait ne plus posséder l'autorité morale requise. Sa décision de se retirer du dossier a donné lieu à une nouvelle plainte contre lui et à la formation d'un comité d'enquête. Bien que le comité d'enquête ait alors considéré qu'il était "inconcevable" que les observations qu'une instance déontologique adresse à un juge pour évaluer sa conduite ou exprimer une préoccupation puissent amener celui-ci à se retirer d'un dossier ${ }^{115}$, cette affaire illustre concrètement les dangers bien réels qui résultent de l'absence d'un encadrement législatif explicite au pouvoir d'un juge ou d'un groupe de juges d'intervenir auprès d'un de leurs pairs pour commenter sa conduite.

En vertu des Procédures, les instances internes du Conseil peuvent aussi recommander que les problèmes relevés à la suite de l'examen d'une plainte soient traités en ayant recours à des services de consultation ou à d'autres mesures correctives ${ }^{116}$. Le consentement du juge visé par la plainte, ainsi que la collaboration de son juge en chef, sont nécessaires à l'application de telles mesures correctives, dont le succès entraîne la fermeture du dossier. La Loi sur les juges ne contient cependant aucune assise permettant au Conseil de recommander de telles mesures correctives ou de contribuer à leur mise en place. Elle n'en définit pas non plus la nature, ni n'en précise les modalités. Une discrétion totale est laissée par les Procédures aux instances internes du Conseil canadien de la magistrature quant aux

2002, reproduite en annexe 2 à ce rapport, le sous-comité avait conclu à un manque flagrant de respect du juge envers plusieurs avocats, à de surprenants écarts de patience du juge, ainsi qu'à un abus de pouvoir du juge portant atteinte à l'image de la magistrature. Le juge visé par les reproches du sous-comité avait appris l'existence de la lettre de désapprobation par l'intermédiaire d'une journaliste.

115. Rapport Boilard (C.E.), à la p. 45 (par. 103).

116. Art. 5.1 b), 5.3, 8.1 b) (pour ce qui est du président ou du vice-président du Comité sur la conduite des juges) et 9.6 c) (pour ce qui est d'un souscomité). Un sous-comité a même exprimé l'avis que l'enquête sur la conduite d'un juge vise moins à punir qu'à proposer des mesures correctives: Décision d'un sous-comité concernant le juge Barakett, 24 juillet 2002. 
recommandations qu'elles peuvent faire à ce sujet et le Conseil luimême n'a pas à les approuver ou à les autoriser.

Bien qu'il offre une voie louable pour permettre de désamorcer la situation créée par le dépôt d'une plainte, ce mécanisme présente cependant des lacunes importantes. Les mesures correctives sont décidées à l'insu du plaignant, qui n'en apprend l'existence qu'une fois qu'elles ont été mises en application ${ }^{117}$. Aucune valeur n'est accordée par les Procédures à l'opinion du plaignant quant à leur suffisance ou leur caractère approprié par rapport au contenu de sa plainte. Étant donné que le maintien de la confiance publique constitue l'objectif ultime du processus de plainte, l'absence de consultation du plaignant dans l'identification de la mesure corrective adéquate paraît peu convaincante. De plus, étant donné que ces mesures sont envisagées avant qu'une décision ne soit prise quant à l'opportunité de tenir une enquête publique, il existe une possibilité réelle que le juge visé par la plainte se sente contraint de les accepter afin d'éviter une enquête publique à propos de sa conduite. Cette façon de procéder présente donc des risques pour son indépendance.

L'intervention disciplinaire du Conseil canadien de la magistrature auprès d'un juge soulève aussi la question du rapport qui s'établit entre le plaignant et le juge en raison du dépôt d'une plainte. À ce sujet, la Cour d'appel fédérale a très clairement établi que "la Loi sur les juges ne confère pas de droits aux justiciables en cas d'inconduite d'un juge" et que le Conseil "n'a pas pour mandat de donner effet aux droits des plaignants ou de leur accorder réparation"118. C'est manifestement sur cette base qu'ont été élaborées les Procédures ${ }^{119}$. Dans cette optique, le

117. L'article 12.2 des Procédures laisse au directeur exécutif la discrétion d'informer le plaignant lorsque le dossier est suspendu en attendant l'application de mesures correctives.

118. Taylor c. Canada (Procureur général), supra note 16, à la p. 38 (par. 82).

119. L'art. 3.4 des Procédures permet même au président ou au vice-président du Comité sur la conduite des juges de poursuivre l'examen d'une plainte malgré qu'elle soit retirée par le plaignant, lorsqu'il considère que l'intérêt 
Les ambiguïtés $d u$

(2008-09) 39 R.D.U.S. processus disciplinaire applicable

plaignant ne fait pas valoir une atteinte personnelle auprès du Conseil; il porte plutôt à l'attention des autorités concernées une situation qu'il considère problématique, en leur demandant de la traiter comme elles jugent approprié de le faire et non en fonction de droits subjectifs qu'il pourrait détenir à l'endroit du juge.

Cette façon de caractériser la plainte peut sembler juste lorsqu'elle fait état d'une situation qui ne concerne le plaignant que de manière indirecte, par exemple une plainte reprochant au juge de s'exprimer publiquement à propos d'un sujet controversé. Par contre, lorsque le plaignant relate une conduite qui l'affecte directement, comme des propos déplacés tenus à son égard ou encore un abus de pouvoir dont il a subi les conséquences, elle occulte l'existence d'un véritable rapport de droit entre le juge et le plaignant. Une immunité de poursuite ${ }^{120}$ est d'ailleurs nécessaire pour éviter au juge les conséquences civiles qui découleraient autrement de ce rapport de droit créé entre le plaignant et lui en raison des événements qui lui sont reprochés. Ce rapport de droit ne cesse pas d'exister du seul fait qu'on considère la conduite du juge sous l'angle déontologique ou disciplinaire, plutôt que sous l'angle de la responsabilité civile ${ }^{121}$, et la qualification juridique d'une plainte d'inconduite judiciaire devrait en tenir compte.

public et la bonne administration de la justice l'exigent. Aucune autre disposition des Procédures n'indique les conséquences du retrait d'une plainte à un stade ultérieur, mais il est vraisemblable que le même principe s'applique à toutes les étapes du processus. À l'inverse, la démission ou la retraite d'un juge priverait le Conseil canadien de la magistrature ou l'une de ses instances de toute compétence pour continuer une enquête déjà commencée : Rapport Marshall (C.E.), à la p. 1 .

120. A propos de l'immunité de poursuite judiciaire, voir : Luc Huppé, Le régime juridique du pouvoir judiciaire, Montréal, Wilson et Lafleur, 2000, aux pp. 101-104.

121. Dans un de ses rapports annuels, le Conseil reconnaissait d'ailleurs l'existence de ce rapport de droit en écrivant que "malgré les craintes que la démarche suscite et les risques de déception qu'elle comporte, il est essentiel que les personnes qui s'estiment lésées par la conduite d'un juge aient un recours": Conseil canadien de la magistrature, Rapport annuel 1996-1997, à la p. 15. 
Il est vrai que la destitution ou la réprimande d'un juge ne peuvent d'aucune façon être considérées comme une réparation privée accordée au plaignant. Elles représentent plutôt une sanction collective, imposée au juge pour garantir l'intégrité de la fonction judiciaire. En ce sens, il semble exact d'affirmer que le processus disciplinaire applicable aux membres de la magistrature n'accorde au plaignant aucun droit à une réparation. Ceci étant, le fait d'être directement affecté par la conduite d'un juge donne au plaignant un intérêt particulier, distinct de celui des autres justiciables, à ce que la conduite du juge fasse l'objet d'un examen public. Certaines attentes légitimes de sa part devraient donc être reconnues; entre autres, le dossier de sa plainte ne devrait pas être fermé sans que l'occasion lui soit donnée de faire des représentations quant aux mesures qui, selon les instances internes du Conseil, rendent inutile la tenue d'une enquête publique.

\section{CONCLUSION}

L'approche restrictive du Conseil canadien de la magistrature dans le traitement des plaintes déposées à l'égard des juges de nomination fédérale trouve une justification de taille dans le texte même de la Loi sur les juges: le rapport remis au ministre de la Justice du Canada par le Conseil vise uniquement à déterminer s'il y a lieu de destituer le juge parce qu'il est inapte à remplir utilement ses fonctions ${ }^{122}$. Ainsi défini de façon extrêmement limitative, le mécanisme d'examen des plaintes établi par la Loi sur les juges accuse cependant un retard manifeste par rapport à l'évolution du droit canadien.

Au moment où le Parlement fédéral a mis ce mécanisme en place, au début des années 1970, l'encadrement juridique de la déontologie et de la discipline judiciaires n'en était encore au Canada qu'à ses premiers balbutiements. Ce domaine a connu

122. Loi sur les juges, supra note 4, art. 65. 
Les ambiguïtés $d u$

(2008-09) 39 R.D.U.S. processus disciplinaire applicable

depuis des développements substantiels ${ }^{123}$. La Cour suprême du Canada a ainsi clairement énoncé qu'en raison des fonctions qu'ils exercent, les juges doivent avoir une conduite exemplaire et que la confiance publique dans les institutions judiciaires en dépend ${ }^{124}$. Elle a aussi établi que la magistrature doit se conformer aux exigences requises par le statut qu'elle occupe dans la société125 et qu'il est donc nécessaire que la conduite des juges soit encadrée par des obligations déontologiques' ${ }^{126}$. De même, la Cour d'appel fédérale a exprimé sans réserve qu'un régime adéquat d'examen de la conduite des juges est essentiel pour préserver la confiance du public dans la magistrature ${ }^{127}$. L'existence d'une responsabilité disciplinaire pour les membres de la magistrature est maintenant considérée comme une modalité indispensable de l'indépendance que la Constitution canadienne leur garantit ${ }^{128}$. Des mesures

123. À ce sujet, voir Luc Huppé, Histoire des institutions judiciaires du Canada, Montréal, Wilson et Lafleur, 2007, aux pp. 630-635 et 736-740.

124. "[...] les qualités personnelles, la conduite et l'image que le juge projette sont tributaires de celles de l'ensemble du système judiciaire et, par le fait même, de la confiance que le public place en celui-ci. [...] La population exigera donc de celui qui exerce une fonction judiciaire une conduite quasiirréprochable. [...] Il devra être et donner l'apparence d'être un exemple d'impartialité, d'indépendance et d'intégrité. Les exigences à son endroit se situent à un niveau bien supérieur à celui de ses concitoyens" : Re Therrien, supra note 20, aux pp. 75-76 (par. 110-111).

125. Id., à la p. 76 (par. 112).

126. La Cour suprême du Canada a ainsi reconnu "[...] la nécessité qu'il existe au sein de la magistrature, des normes de conduite conçues pour soutenir la confiance que place le justiciable en cette dernière et ceci, pour assurer la permanence de la règle de droit telle qu'elle s'exprime aujourd'hui.": Ruffo c. Conseil de la magistrature, supra note 7, p. 332 (par. 109). Un comité d'enquête a exprimé l'opinion que "le public a droit à une procédure méthodique d'examen des plaintes portées contre les juges des cours supérieures": Décision $d u$ comité d'enquête en vertu des paragraphes 63 (2) et 63 (3) de la Loi sur les juges relativement à M. le juge F.L. Gratton de la Cour de l'Ontario (division générale), 26 janvier 1994, à la p. 19.

127. Cosgrove, supra note 19, à la p. 729 (par. 32).

128. Plusieurs auteurs ont déjà émis des opinions allant en ce sens : "Les notions d'indépendance et de déontologie judiciaires sont interdépendantes. Sans déontologie, l'indépendance ne se justifie pas. Sans indépendance, la déontologie aujourd'hui ne suffit pas" (Glenn, supra note 64, à la p. 303); "[...] l'indépendance judiciaire ne se conçoit 
concrètes sont essentielles pour conférer une portée réelle à ces principes.

Il est significatif de constater que le cadre établi par la Loi sur les juges à l'époque de la création du Conseil canadien de la magistrature est demeuré quasiment inchangé en ce qui a trait au processus de traitement des plaintes. Une conséquence importante découle de cette constatation: la perspective dans laquelle la Loi sur les juges invite le Conseil canadien de la magistrature à traiter les plaintes formulées contre les juges de nomination fédérale ne concorde plus avec la perspective dans laquelle le droit canadien rend dorénavant les juges redevables de leur conduite. Cette évolution du droit met plus particulièrement en relief l'insuffisance de la Loi sur les juges relativement aux inconduites judiciaires mineures. Sans la compétence explicite d'imposer des sanctions proportionnées aux inconduites qu'il constate, le Conseil canadien de la magistrature ne peut accomplir sa "mission de veiller au respect de la déontologie judiciaire»129. Faute d'un instrument législatif plus fin, la compétence d'enquête du Conseil ne peut servir à mettre en œuvre, dans l'ensemble des situations où elles pourraient potentiellement trouver application, les obligations déontologiques des juges de nomination fédérale.

En consultation avec la magistrature, le Parlement fédéral a donc le devoir constitutionnel de mettre la Loi sur les juges au diapason du cadre juridique contemporain ${ }^{130}$. Maintes fois les

plus de nos jours sans une obligation pour les juges de rendre compte de leur conduite judiciaire et extrajudiciaire" (Yves-Marie Morissette, "Comment concilier déontologie et indépendance judiciaires?", (2003) 48 R.D. McGill 297, p. 302); "[...] real accountability requires that judicial councils have access to more than the ultimate sanction of removal" (A. Wayne Mackay, "Judicial Free Speech and Accountability: Should Judges Be Seen but Not Heard?», (1993) 3 R.N.D.C. 159, à la p. 210).

129. Rapport Bienvenue (C.E.), à la p. 58.

130. Bien que la compétence accordée au Parlement fédéral par l'article 101 de la Loi constitutionnelle de 1867 à l'égard des juges de nomination fédérale ne concerne que leur rémunération, la jurisprudence laisse entrevoir à ce sujet une plus large compétence législative fédérale que celle expressément énoncée dans les textes constitutionnels. Ainsi, il 
Les ambiguïtés du

(2008-09) 39 R.D.U.S. processus disciplinaire applicable

aux juges de nomination fédérale

tribunaux ont-ils répété que la confiance des justiciables dans leurs institutions judiciaires représente une condition indispensable au maintien de la primauté du droit. La réalisation de cet objectif ne dépend pas uniquement de la droiture et des capacités des membres de la magistrature. Les institutions législatives doivent elles aussi participer à la préservation de la confiance publique envers les tribunaux, non seulement parce qu'elles détiennent seules le pouvoir d'établir des structures contraignantes, mais aussi en raison de la valeur de transparence et d'exemplarité propre au texte législatif. Pour que les justiciables puissent pleinement adhérer à leur système juridique, il est indispensable que la loi exprime le consensus social qui fonde la légitimité des institutions judiciaires. Une législation moderne, en phase avec le contexte juridique actuel, contribuerait mieux au maintien de leur confiance que l'actuelle Loi sur les juges.

semble que les tribunaux seraient disposés à considérer que les questions reliées au statut, à l'indépendance et aux fonctions judiciaires des juges de nomination fédérale, y compris les mécanismes permettant de traiter des plaintes contre ces juges et d'enquêter relativement à leur conduite, échappent à la compétence provinciale : Mackeigan c. Hickman, [1989] 2 R.C.S. 796, aux pp. 811-813. Bien que ces propos aient été tenus dans le cadre d'une dissidence, ils ont ensuite été repris par la Cour fédérale dans Gratton, supra note 34, aux pp. 796-797. 


\section{ANNEXE : LISTE DES RAPPORTS D'ENQUÊTE'131}

Trois rapports d'enquête datant de la période où les comités d'enquête procédaient en privé n'ont pas été publiés ${ }^{132}$.

Report of the Committee of Investigation to the Canadian Judicial Council into the Conduct of the Hon. Mr. Justice Berger, 31 mai 1982 (appelé le "Rapport Berger (C.E.)»)133; ce rapport a été partiellement désavoué par une majorité de membres du Conseil : Résolution du Conseil canadien de la magistrature, 31 mai $1982^{134}$.

Rapport au Conseil canadien de la magistrature déposé par le comité d'enquête nommé conformément aux dispositions du paragraphe 63 (1) de la Loi sur les juges à la suite d'une demande du Procureur général de la Nouvelle-Écosse, 27 août 1990 (appelé le "Rapport Marshall (C.E.)»)135; ce rapport a été entériné à l'unanimité par le Conseili36.

Rapport au Conseil canadien de la magistrature du comité d'enquête nommé conformément aux dispositions du paragraphe 63 (1) de la Loi sur les juges pour mener une enquête publique relativement à la conduite de M. le juge Jean Bienvenue de la Cour supérieure du Québec dans la cause La Reine c. Théberge, juin 1996 (appelé le "Rapport Bienvenue (C.E.)") ${ }^{137}$; ce rapport a été entériné par une majorité de membres du Conseil : Rapport $d u$

131. Les rapports d'enquête rendus depuis les années 1990, ainsi que quelques rapports du Conseil au ministre de la Justice du Canada, sont disponibles en ligne au site Internet du Conseil canadien de la magistrature : http://www.cjc-ccm.gc.ca/.

132. Leur contenu est résumé dans le Rapport annuel du Conseil canadien de la magistrature, 1996-1997, aux pp. 19-20.

133. Publié à (1983) 28 R.D. McGill 382.

134. Id., à la p. 379; voir aussi : Conseil canadien de la magistrature, Rapport annuel 1996-1997, à la p. 20.

135. Aussi publié à : (1991) 40 R.D. U.N.-B. 210.

136. Rapport annuel du Conseil canadien de la magistrature, 1990-1991, à la p. 13.

137. Aussi publié à : (1997-1998) 9 R.N.D.C. 357. 
Les ambiguïtés $d u$

(2008-09) 39 R.D.U.S. processus disciplinaire applicable

113

aux juges de nomination fédérale

Conseil canadien de la magistrature au ministre de la Justice aux termes du paragraphe 65 (1) de la Loi sur les juges concernant les plaintes au sujet du comportement de Monsieur le juge Jean Bienvenue de la Cour supérieure du Québec dans la cause $R$. c. Théberge, 20 septembre 1996 (appelé le "Rapport Bienvenue (C.C.M.)川138.

Rapport au Conseil canadien de la magistrature du comité d'enquête nommé conformément au paragraphe 63 (1) de la Loi sur les juges pour mener une enquête sur le juge Bernard Flynn relativement aux propos tenus par celui-ci à une journaliste dont l'article a paru dans le journal Le Devoir du 23 février 2002, 12 décembre 2002 (appelé le "Rapport Flynn (C.E.)»); ce rapport a été entériné à l'unanimité par le Conseil : Rapport présenté par le Conseil canadien de la magistrature au ministre de la Justice du Canada conformément au paragraphe 63 (1) de la Loi sur les juges concernant la conduite de M. le juge Bernard Flynn de la Cour supérieure du Québec, 25 mars $2003^{139}$.

Rapport au Conseil canadien de la magistrature du Comité d'enquête nommé conformément au paragraphe 63 (3) de la Loi sur les juges pour mener une enquête sur le juge Jean-Guy Boilard relativement à la décision qu'il a prise le 22 juillet 2002 d'abandonner la gestion du procès R. c. Beauchamp, 5 août 2003 (appelé le "Rapport Boilard (C.E.)»); ce rapport a été partiellement désavoué par le Conseil, à l'unanimité: Rapport du Conseil canadien de la magistrature présenté au ministre de la Justice du Canada en vertu de l'art. 65 (1) de la Loi sur les juges et concernant le juge Jean-Guy Boilard de la Cour supérieure du Québec, 19 décembre 2003 (appelé le "Rapport Boilard (C.C.M.)").

Rapport au Conseil canadien de la magistrature du comité d'enquête constitué en vertu du paragraphe 63 (3) de la Loi sur les juges pour enquêter sur la conduite du juge Theodore Matlow de la

138. Publié dans le Rapport annuel du Conseil de la magistrature, 1996-1997, à la p. 69.

139. Publié dans le Rapport annuel du Conseil de la magistrature, 2002-2003, à la p. 65. 
Cour supérieure de justice de l'Ontario, 28 mai 2008 (appelé le "Rapport Matlow (C.E.)"); la recommandation de destitution formulée par ce rapport a été mise de côté par le Conseil : Rapport du Conseil canadien de la magistrature au ministre de la Justice concernant la conduite de l'honorable T. Matlow, 3 décembre 2008 (appelé «Rapport Matlow (C.C.M.)»).

Rapport au Conseil canadien de la magistrature du comité d'enquête constitué en vertu du paragraphe 63 (3) de la Loi sur les juges pour enquêter sur la conduite du juge Paul Cosgrove de la Cour supérieure de justice de l'Ontario, 27 novembre 2008 (appelé le "Rapport Cosgrove (C.E.)»); la recommandation de destitution formulée dans ce rapport a été entérinée par le Conseil : Rapport du Conseil canadien de la magistrature au ministre de la Justice concernant la conduite de l'honorable Paul Cosgrove, 30 mars 2009 (appelé «Rapport Cosgrove (C.C.M.)»). 